\title{
Discours
}

Revue de linguistique, psycholinguistique et informatique. A journal of linguistics, psycholinguistics and computational linguistics

13 | 2013

Varia

\section{Temporalité et compétences (méta)linguistiques}

\section{Emmanuelle Roussel}

\section{(2) OpenEdition}

\section{Journals}

Édition électronique

URL : http://journals.openedition.org/discours/8846

DOI : $10.4000 /$ discours.8846

ISSN : 1963-1723

Éditeur :

Laboratoire LATTICE, Presses universitaires de Caen

\section{Référence électronique}

Emmanuelle Roussel, «Temporalité et compétences (méta)linguistiques », Discours [En ligne],

13 | 2013, mis en ligne le 23 décembre 2013, consulté le 01 mai 2019. URL : http://

journals.openedition.org/discours/8846 ; DOI : 10.4000/discours.8846

\section{(c) $($ i) $(9)$}

Discours est mis à disposition selon les termes de la licence Creative Commons Attribution - Pas d'Utilisation Commerciale - Pas de Modification 4.0 International. 

Revue de linguistique, psycholinguistique et informatique

\section{Temporalité et compétences (méta)linguistiques}

Emmanuelle Roussel

CRISCO, EA 4255

Université de Caen Basse-Normandie

Emmanuelle Roussel, «Temporalité et compétences (méta)linguistiques», Discours [En ligne], 13 | 2013, mis en ligne le 23 décembre 2013.

URL: http://discours.revues.org/8846

Titre du numéro: Varia

Coordination: Stéphane Ferrari et Christian Raymond 



\title{
Temporalité et compétences (méta)linguistiques
}

Emmanuelle Roussel

CRISCO, EA 4255

Université de Caen Basse-Normandie

\begin{abstract}
La présente étude menée sur les enfants d'une classe de cours préparatoire (CP) repose les deux questions centrales de la primauté de l'aspect et de la fonction exacte des flexions verbales en les intégrant dans une perspective biolinguistique de l'acquisition du langage. Cet angle d'étude permet ainsi d'avancer une explication à la dichotomie observée entre la régularité syntaxique observée en $\mathrm{CP}$ et la conscience, a priori vague à cet âge, de la temporalité. L'enquête démontre que les enfants manipulent effectivement plusieurs formes temporelles ainsi que les notions d'antériorité, de postériorité et de succession. En revanche, ces formes ne semblent pas tant refléter des valeurs aspectuelles que des valeurs perceptives d'une temporalité propre à chacun de ces enfants.
\end{abstract}

Mots clés: acquisition du langage, cognition, langue parlée, temps verbaux

This paper focuses on how French first-year primary school pupils deal with tensed verb forms, at an age when they are thought to have not yet developed a sense of time. Indeed, it is assumed that up to the age of six or seven children assign aspectual functions to tense inflections even if they build these forms correctly. The hypothesis behind the study is that this dichotomy is to be linked to various physiological properties inherent in language acquisition. These tensed verbal forms consequently appear to take on a perceptual function rather than an aspectual one. It is therefore a safe assumption that children acquire their sense of time through perceptual properties.

Keywords: language acquisition, cognition, spoken language, tenses

\section{Introduction}

Le but de cet article est d'évaluer la compétence linguistique d'enfants francophones scolarisés âgés de 6 à 8 ans (niveau cours préparatoire), qui partagent une compétence métalinguistique sur le temps (Droit-Volet, 20II : 152), thème a priori ambigu dans la mesure où la lexie «temps» peut en français dénoter le temps grammatical, le temps extralinguistique ou encore le temps atmosphérique. Les trois notions associées à ces sémantismes se construisent différemment selon que le point de vue adopté est linguistique ou non (c'est-à-dire extralinguistique, physiologique, chronobiologique, mémoriel), ce qui conduit à une analyse croisée des phénomènes. Partant, si les formes temporelles constituent un vecteur privilégié de l'expression linguistique de la temporalité, elles ne sont qu'un moyen parmi d'autres susceptibles de refléter la compréhension des phénomènes temporels de la part des enfants. Dans la mesure où ceux-ci ne sont pas censément aptes à concevoir le concept de temps avant l'âge de 7 ans (Piaget, 1937 et 2006 [1926]; Fuchs et al., 1978a: 30-31 et 1978b: 24-25; Droit-Volet, 200I: 34; Izard et al., 20II : 322, 324 et 327-328), nous avons souhaité tenter de préciser quelle acception est privilégiée par les enfants en phase "prétemporelle», autrement dit vers 6 ans et demi (en théorie), à partir de la polysémie du terme «temps». L'enquête 
que nous avons menée illustre une étude transdisciplinaire de la temporalité qui porte, entre autres, sur le statut du temps et de l'aspect (Roussel, 2009).

L'étude vise ainsi à apporter quelques éléments explicatifs à la primauté de l'aspect, qui est soit décrite dans l'évolution de la langue (Benveniste, 1976 [1966] : 173; Slobin, I97I ; Ferreiro, 197I ; Bronckart, 1976; Kurylowicz, 1977; Fuchs et al., 1978a:30-31 et 1978b : 24-25; Haudry, 1979: 79; Sergent, 2005: 173; Fortson, 2010: 91 et I05; Vet, 20II : 464-465) comme du langage (Berko, 1958; Ferreiro, 1971; Ferreiro et Sinclair, I97I ; Bronckart, I976; Slobin, I97I, 198I, 1985, I99I et 1996; Bickerton, I974 et I98I ; Andersen, 1986 et 1990 ; Andersen et Shirai, 1994 et 1996; Robison, 1990; King, 2006; Dehaene-Lambertz, 2008), soit remise en cause (Labeau, 2002, 2003, 2005a et $2005 \mathrm{~b})$. Trois principaux travaux qui concernent l'anglais et le français, et soulignent certaines caractéristiques syntaxiques et sémantiques des formes temporelles au cours de la phase de leur acquisition par l'enfant, servent de support à une confrontation aux récentes données biocognitives sur le sujet. Pour ce faire, nous avons interrogé les enfants d'une classe de CP en sessions individuelles de io minutes.

\section{Caractéristiques syntaxiques et sémantiques des formes temporelles chez l'enfant}

Les éléments sur lesquels repose notre enquête sont de trois ordres: syntaxique, aspectuel et temporel. Ils concernent la syntaxe du révolu chez l'enfant anglais de niveau CP (Berko, 1958; Ullman, 2006), la prise en considération de l'aspect interne des verbes par les enfants francophones avant qu'ils ne soient capables de concevoir la notion même de temps (Ferreiro, 1971; Fuchs et al., 1978a et 1978b; Smith, 1980; Weist, 1986 et 1989), ainsi que l'application d'une fonction temporelle à ces mêmes formes verbales par l'enfant francophone (Bronckart, 1976 ; Clark, $1985^{1}$ ). Quelle que soit la position adoptée quant au statut de l'aspect (sa nature semble clairement relever de la lexie verbale, autrement dit l'aspect évoqué est l'aspect lexical, ou interne au lexème verbal, au point que Bickerton [1981 et 1984] évoque une aptitude génétique à différencier les aspects lexicaux ${ }^{2}$ ), ces éléments mettent en évidence la présence d'une régularité syntaxique relative au temps grammatical chez l'enfant de la tranche d'âge concernée, avant que celui-ci n'ait, semble-t-il, acquis la notion d'écoulement du temps. Par conséquent, lesdits éléments posent essentiellement deux questions: celle du sémantisme réel affecté à ces formes (et, par suite, celle du rôle véritable de la désinence temporelle), ainsi que celle de la manière dont se construit ce sémantisme dans une perspective qui prend en compte le phénomène plus large de l'acquisition du langage.

Les sources citées font coïncider le niveau scolaire des enfants concernés par l'enquête - CP/ 6 ans - avec le début théorique de l'acquisition de la notion de temps.

1. Pour qui les enfants francophones utilisent les temps du passé pour dénoter le résultat et non pour encoder le caractère révolu de l'action nommée par le verbe.

2. Même si pour Bloom et al. (1980) les deux types d'aspect (lexical et grammatical) importeraient dans le marquage temporel. 
C'est en effet l'âge des enfants observés par Berko, qui s'est précisément intéressée au statut et au mode d'acquisition de la désinence -ed du prétérit dit «régulier» ${ }^{3}$ de l'anglais (voir également Leonard, 1989; Pierce, 1992; Meisel, 1992; Leonard et al., 1992; Chomsky, 1993; Rice et Oetting, 1993; Clahsen et al., 1993; Rizzi, I993; désinence -ed y compris considérée comme marqueur clinique en tant que point d'achoppement chez les enfants souffrant de troubles spécifiques du langage: Marchman et Weismer, 1994; Tomblin, 1994; Ullman et Gopnik, 1994; Bishop, 1994; King et al., 1995; Oetting et al., 1995; Rice et Wexler, 1996; McClelland et Patterson, 2002). Anglophones, ces enfants n'en illustrent pas moins des régularités flexionnelles, relevées chez les enfants francophones, que l'on peut résumer en termes de «surgénéralisation des règles de grammaire» (Dehaene-Lambertz, 2008: 169 ${ }^{4}$ ). Le phénomène en question semble donc transcender les langues (Pierce, 1992), d'où notre mention de l'étude de Berko. Les enfants observés par Ferreiro et Bronckart étaient, quant à eux, un peu plus jeunes, l'organisation spécifique de ces études consistant alors à demander aux enfants concernés de décrire une situation mimée par des poupées. Nous avons, pour notre part, interrogé directement les enfants en prêtant attention à leur gestion des formes temporelles.

\section{1. Étude menée par Berko (1958)}

Pour mémoire, dans son étude intitulée «The Child's Learning of English Morphology», Berko (1958: 150-177) a tenté de mesurer l'aptitude des enfants à créer des prétérits (ou des pluriels) sur le modèle de formes existantes telles que laughed ou birds, à partir d'éléments dépourvus de sens, comme par exemple le nom ${ }^{*}$ gutch (Berko, 1958: 150). En ce qui concerne le prétérit, elle a observé que les enfants étaient d'abord capables d'appliquer la règle qui consiste à ajouter les alvéolaires sonore /-d/ ou sourde /-t/ à des occurrences verbales «non connues» comme ${ }^{*}$ bing, ${ }^{*}$ gling, ${ }^{*}$ rick, ${ }^{*}$ bod. En revanche, ils ne savaient pas encore ajouter la désinence complète (Berko, 1958: 165). Lorsqu'ils connaissaient la forme complète $(\checkmark$ melted $)$, ils ne parvenaient pas à la généraliser. Les formes irrégulières, quant à elles, n'étaient pas maîtrisées. Ce point de vue sera plus tard remis en question par Bybee (1991) notamment, qui privilégiera une conception lexicaliste du phénomène d'acquisition (Noyau, 200I: 182). Cependant, la présente étude, qui se focalise sur l'acquisition des flexions verbales, permet de s'interroger à nouveau sur le statut flexionnel, à la suite de Rumelhart et McClelland (1986), qui ont montré que le prétérit des verbes irréguliers anglais relève d'une combinaison flexionnelle dans la mesure où nombre de ces verbes contiennent bien une désinence $-d$ ou $-t$, et ce, même si ces verbes sont stockés dans la mémoire sémantique.

3. Rappelons que les termes «régulier» et «irrégulier» renvoient à la conjugaison, et ne sont aucunement synonymes des termes «faible» et «fort». En effet, de nombreux verbes à conjugaison «irrégulière» sont des verbes faibles sur le plan diachronique.

4. D'où la création de constructions agrammaticales, qui concernent aussi bien les flexions nominales que verbales, telles que: «* Les petits garçons prendaient des bonbons» ou «* Les chevals sontaient partis». Elles correspondent en anglais à une utilisation massive de la désinence - $e d$ et de la désinence $-s$ pour former le prétérit et le pluriel respectivement, y compris sur les verbes et les noms à fonctionnement dit «irrégulier». 
Berko a tiré deux conclusions de ses travaux. D'une part, que les formes régulières se mettaient en place d'abord sous forme allomorphique, puis sous forme pleine, et ce, bien avant les formes irrégulières. D'autre part, que la primauté des formes régulières prouvait que la connaissance de la langue consistait en autre chose que le stockage de données mémorisées, sinon, toujours selon elle, les enfants auraient refusé de répondre à ses questions dès lors qu'elles traitaient d'une lexie qu'ils n'avaient jamais entendue, telle *wug (Berko, 1958: 170).

Ces remarques relèvent, entre autres, de la dichotomie de fonctionnement des mémoires afférentes à l'acquisition du langage, que nous ne développerons pas dans ces lignes. Nous renvoyons à la littérature dédiée à cette question, de près (Rumelhart et McClelland, I986; Pinker, 1991, 1999 et 200I; Desmarais et al., I997; Rice et al., 2000; McClelland et Patterson, 2002; Ullman, 2006; Dehaene, 2006 et 2008; Dehaene-Lambertz, 2006; Vincent, 2007; Knierim, 2007; Roussel, 2007a; Camos et al., 2010; Mora et al., 2010; Siaud-Facchin, 2012) ou de loin (Ramscar, 2002; Cotte, 2008: 22-23). Nous mentionnerons simplement le fait que cette dichotomie a directement trait aux propriétés de l'aire de Broca et des mémoires procédurale et déclarative qui se répartissent la gestion de la syntaxe (aire de Broca et mémoire procédurale) et du lexique (mémoire déclarative) dans le cerveau. La dichotomie en question est on ne peut plus pertinente pour ce qui est de l'émergence des formes de temps et d'aspect selon que la conjugaison du verbe est régulière ou non (Myhill, I99I; Noyau, 200I: I83), puisque cette opposition illustre la propriété du cerveau à traiter les conjugaisons irrégulières non plus comme des verbes mais comme du lexique - précisément parce qu'elles sont irrégulières - et, partant, plus rapidement que les conjugaisons régulières. En effet, et de manière inattendue, apprendre une conjugaison irrégulière nécessite un travail moindre sur le plan cérébral que celui qui consiste à ajouter une désinence à une base verbale (Ullman, 2006; Roussel, 2007a). On rejoint le fonctionnement des ordinateurs sur ce point. Ce serait donc prioritairement en raison de leur conjugaison que ces formes sont traitées "comme» du lexique, et non en raison de leur catégorisation première en tant que lexique. Cependant, la capacité qui consiste à ajouter une désinence, et qui relève d'un traitement séquentiel des éléments lié au phénomène plus large de la planification (propriété cognitive fondamentale de l'être humain - Coppens, 2003), est en place plus tôt sur le plan cérébral (Dehaene-Lambertz, 2006; Vincent, 2007) que celle liée à l'apprentissage par cœur du lexique, qui est à maturation vers l'âge de 7 ans. Autant d'éléments qui soulignent la nécessité de prendre en compte les données cognitives récentes dans l'analyse linguistique de la temporalité.

\section{2. Études menées par Ferreiro (1971) et Bronckart (1976)}

L'étude effectuée par Ferreiro portait sur 130 enfants francophones âgés de 2 à 4 ans. Elle a cherché à comprendre dans quelle mesure ces enfants intégraient l'ordre d'occurrences d'événements dénotés par des verbes conjugués à des temps différents. Les rapports de succession ou de simultanéité entre événements étaient là en question. Ses observations l'ont conduite à poser que, du point de vue de 
l'enfant, le temps sur les verbes ne renvoyait aucunement à l'ordre des événements. L'alternance entre le présent et l'imparfait semblait ainsi relever de considérations aspectuelles plutôt que servir à dénoter des rapports temporels (Ferreiro, 1971 : 39). Bronckart, pour sa part, s'est concentré sur des enfants de 6 ans qui étaient en théorie censés ne plus appliquer cette fonction aspectuelle aux temps verbaux du français. En effet, avant 6 ans, l'enfant «semble attribuer un taux de résultativité à chaque action en interprétant cette action en termes aspectuels» (Bronckart, 1976: 62). C'est-à-dire que les actions dotées d'un résultat clair étaient principalement décrites au passé composé, celles qui n'avaient pas de résultat «observable» ${ }^{5}$ l'étaient au présent (Bronckart, 1976: 96). Pour les enfants ayant entre 3 et 6 ans, le présent et l'imparfait avaient, quant à eux, une utilisation parallèle à l'accroissement de la durée (Bronckart, 1976: 99). Par conséquent, l'âge de 6 ans semblait délimiter la naissance de la fonction temporelle des temps du verbe; et à partir de ce moment-là, Bronckart a observé que le choix de la flexion dépendait du délai de production. Si l'action était terminée depuis 2 secondes, l'enfant avait tendance à employer un présent (le temps du verbe traduisait ainsi une simultanéité temporelle); si le délai entre la fin de l'action et la prise de parole passait à 7 secondes, l'enfant employait le passé composé, qui était maintenu lorsque le délai s'inscrivait entre 7 et 25 secondes (avec un signifié temporel de l'ordre de l'antériorité). Bronckart de conclure alors qu'entre 3 et 5 ans, les flexions dépendaient de la caractéristique aspectuelle de durée. Entre 5 et 6 ans, ce paramètre semblait moins déterminant. Ensuite, il était entièrement indépendant de l'intervalle spatiotemporel. On peut également se référer à Labelle (1994: IO4).

Nous avons, de notre côté, souhaité nous faire notre propre idée auprès d'enfants de niveau $\mathrm{CP}$, en cherchant à mesurer la part vouée à la métalangue et celle vouée à la langue dans la gestion des notions aspectuelle et/ou temporelles en situation. Avec une hypothèse de départ négative pour ce qui est du temps: puisque l'enfant n'en a pas acquis la notion sur le plan physiologique, il ne peut en avoir conscience ni, a fortiori, en parler. Mais a-t-il la notion de ce qui se traduit par une perception aspectuelle des événements? Comment réagit-il aux formes temporelles? Enfin, au cas où il aurait une notion de temps, est-il envisageable de préciser quelle en est la nature?

\section{Données de l'enquête}

Nous avons interrogé I8 enfants d'une classe de CP entre le début du mois de mai et la fin du mois de juin 2008. À l'évidence, le nombre réduit d'enfants privilégie une évaluation d'ordre qualitatif qu'une enquête à plus grande échelle permettrait de confirmer ou d'infirmer. Les 8 filles et les io garçons formaient 3 groupes en fonction de leur âge, dont voici une répartition détaillée:

5. Ce qualificatif justifie le choix que nous faisons de partir des conclusions de Bronckart pour notre étude, qui se focalise sur les modalités de perception visuelle. 


\begin{tabular}{|c|c|c|c|}
\hline Groupe 6 (Io enfants) & Année & Mois & Jours \\
\hline I & 6 & o & o \\
\hline 2 & 6 & 3 & I2 \\
\hline 3 & 6 & 3 & 26 \\
\hline 4 & 6 & 6 & ० \\
\hline 5 & 6 & 6 & 23 \\
\hline 6 & 6 & 7 & I \\
\hline 7 & 6 & 7 & 15 \\
\hline 8 & 6 & 8 & 3 \\
\hline 9 & 6 & 8 & I4 \\
\hline IO & 6 & 9 & 8 \\
\hline Groupe 7 ( 7 enfants) & Année & Mois & Jours \\
\hline I & 7 & $\mathrm{I}$ & ० \\
\hline 2 & 7 & I & 5 \\
\hline 3 & 7 & I & I8 \\
\hline 4 & 7 & 3 & 9 \\
\hline 5 & 7 & 4 & $\mathrm{I} 2$ \\
\hline 6 & 7 & 7 & 6 \\
\hline 7 & 7 & II & 6 \\
\hline Groupe 8 (I enfant) & Année & Mois & Jours \\
\hline I & 8 & 3 & 7 \\
\hline
\end{tabular}

Tableau A. Âge précis des enfants de l'enquête

On observe d'emblée un écart de 2 ans et 3 mois entre l'enfant le plus jeune et le plus âgé. Il se trouve que cet écart correspond à la période théorique d'acquisition de la notion de temps évoquée plus haut, d'où notre choix de ce niveau qui a priori ne cultive la mémorisation ni de la notion de temps ni des désinences ou conjugaisons en tant que telles, et où ce qui peut être observé est au plus près des capacités de chaque enfant, étant donné que dans le système scolaire français, ce travail ciblé et explicite sur les temps grammaticaux est effectué l'année suivante, en cours élémentaire $\mathrm{I}^{\mathrm{re}}$ année ( $\left.\mathrm{CEI}\right)$. En accord avec l'enseignante de la classe concernée, les séances se sont déroulées en fin de journée, après la récréation de l'après-midi, autour d'un formulaire de 14 questions dont la dernière consistait à demander aux enfants de dessiner «le temps» («Veux-tu me dessiner le temps?»), 
afin de vérifier si le dessin laissait transparaître des éléments de réponse que l'enfant n'avait pas su ou pu formuler ${ }^{6}$.

\section{Enquête}

\subsection{Questionnaire}

Tout en partant du principe que l'absence de réponse était significative (voire requise a priori) pour l'étude, le questionnaire avait pour objectif de cibler la «conscience temporelle» des enfants comme indiqué plus haut, et de la rapporter à leur métalangue. C'est la raison pour laquelle des questions fermées ont d'emblée introduit le thème de la séance, suivies de questions ouvertes nécessitant une mise en mots de la réponse par l'enfant, puis de la dernière question fermée portant sur le dessin.

Les formes temporelles de ces questions reprenaient celles sur lesquelles porte l'étude, afin de voir si les enfants «entendaient» ces formes. Ainsi, une même proposition a été utilisée plusieurs fois, la conjugaison seule étant modifiée, pour que les enfants ne soient pas perturbés par l'effort lié à la compréhension de nouveaux lexèmes et puissent se concentrer sur le verbe. Les notions évoquées étaient alors fédératrices ou faisaient partie de l'univers type des enfants ${ }^{7}$.

En vue d'obtenir une réponse liée à la temporalité, certaines questions ont été construites sur l'adverbe interrogatif «quand». Et en vue d'obtenir une réponse justifiant un lien à la temporalité, d'autres ont été construites sur l'adverbe interrogatif «pourquoi». Dans une perspective de non-blocage de l'échange, la quasi-totalité des questions a reproduit le français parlé informel («C'est quand: W?»; «Pourquoi je dis $\mathrm{X}$ ?»). Les questions fermées en revanche ont respecté l'inversion verbe sujet («Sais-tu ce qu'est Y?»; «Peux-tu me dire Z?»).

Les questions, dont les différentes notions temporelles relevaient aussi bien du lexique que de la conjugaison, sont répertoriées ci-dessous:

[I] Sais-tu ce qu'est le temps?

[2] Peux-tu me dire ce que c'est?

[3] Peux-tu me dire à quoi ça sert?

[4] Comment fait-on pour parler du temps?

6. Nous avons parallèlement eu la possibilité d'interroger de manière isolée quelques enfants plus âgés en classe de $\mathrm{CE}_{\mathrm{I}}$ (7 ans et 28 jours; 7 ans, 9 mois et 20 jours) ou de CE2 ( 8 ans, 6 mois et 5 jours). Leur faible nombre empêche toute étude statistique des réponses obtenues; elles renseignent cependant sur l'évolution des capacités d'élaboration de ces enfants.

7. /Jouer à la poupée et/ou au ballon/ (à la Game Boy pour les plus grands), /manger des frites/ (pour tous). 
[5] C'est quand: «Je joue à la poupée/au ballon»?

[6] C’est quand: «J'ai joué à la poupée/au ballon»?

[7] C’est quand: «Je jouais à la poupée/au ballon»?

[8] C'est quand «hier»?

[9] C’est quand «demain»?

[Io] Pourquoi je dis (la phrase): «Je mange des frites»? (peut-on dire autre chose?)

[II] Pourquoi je dis (la phrase): «J'ai mangé des frites»? [...]

[I2] Pourquoi je dis (la phrase): «Je mangeais des frites»? [...]

[13] Pourquoi je dis (la phrase): «J'avais mangé des frites»? [...]

[I4] Veux-tu me dessiner le temps?

\subsection{Description}

\subsubsection{Questions}

Il est d'emblée possible d'indiquer que, bien que les questions pourraient a priori sembler difficiles pour des enfants de cette classe d'âge, tous ont répondu à l'ensemble de ces questions. Sur les i8 questionnaires,

- I enfant $\left(5,5 \% ; 7_{\operatorname{armoj}}^{8}\right)$ a répondu à toutes les questions;

- 2 enfants (II,II $\% ; 6 a 3 m ı$ j et $6 \mathrm{a} 6 \mathrm{~m} 23 \mathrm{j}$ ) ont répondu qu'ils ne savaient pas («Ché pas»; «Je sais pas»; «J'en sais rien») I fois;

- I enfant $(5,5 \%$; 7azm9j) a montré son ignorance en commençant sa réponse par «Parce que» («Parce que... je sais pas»);

- 5 enfants $(27,77 \%$; 6a6m?j, 6a7mij, 6a7mı $5 \mathrm{j}$, 7a7m6j et $8 \mathrm{azm} 7 \mathrm{j})$ ont répondu «Je sais pas» 2 fois;

- 3 enfants (ı6,66 \% ; 6a9m8j, 7aım5j et 7aıım6j) ont répondu «Je sais pas» 3 fois;

- 6 enfants $(33,33 \%)$ ont exprimé leur ignorance plus de 3 fois:

- I enfant (ı6\%; 6азm26j) l'a fait 4 fois;

- 3 enfants ( $50 \%$; 6a8mi4j, 7aimi8j et $7 \mathrm{a} 4 \mathrm{mi} 2 \mathrm{j}$ ) l'ont fait 5 fois;

- I enfant (ı6\%; 6a) l'a fait 6 fois;

- I enfant (ı6\%; 6a8m3j) l'a fait 8 fois.

8. Pour 7 ans, I mois et $\mathrm{o}$ jour. 
Nous avons, à ce stade, pu émettre une première conclusion: il ne semble pas exister de corrélation directe entre l'âge et l'incapacité à répondre de manière satisfaisante («non-réponse»). À chaque extrémité de l'éventail (I fois [5,5\%]- 8 fois [44,4 \%]), on trouve un enfant de 6 ans (6azmı $\mathrm{j}$ : I fois; $6 \mathrm{a} 8 \mathrm{~m} 3 \mathrm{j}: 8$ fois). Il apparait qu'un seul enfant a répondu à 100 \% des questions, tandis que io enfants (55,55\%) n'ont pas su répondre entre I et 3 fois, et que 6 enfants n'ont pas su répondre plus de 3 fois. Autrement dit, I7 enfants (94,44\%) ont éprouvé des difficultés à apporter une réponse claire à la totalité des questions, statistiques qui illustrent la difficulté pressentie lors de l'élaboration de l'enquête. Le nombre assez élevé d'occurrences de non-réponse est logique chez l'enfant de $6 \mathrm{a} 8 \mathrm{~m} 3 \mathrm{j}$. Le nombre élevé de réponses chez l'enfant de 6azmızj l'est moins. Il est intéressant de noter que l'enfant qui a répondu à toutes les questions était à peine plus âgé que l'un de ceux qui «n'a pas su» 5 fois (7armoj contre 7 armi8j). Les 9 enfants qui ont répondu au plus grand nombre de questions ${ }^{9}$ étaient paradoxalement plus jeunes par rapport au groupe classe. 5 appartenaient au groupe 6, 3 au groupe 7 , le dernier étant l'enfant le plus âgé.

Si à présent nous observons la nature des questions qui ont conduit à une réponse du type «Je sais pas», nous remarquons que l'ignorance des enfants a été exprimée 8 fois en réponse à une question ouverte et 6 fois en réponse à une question fermée. Chaque enfant n'a pas su répondre à un type récurrent de question: soit ouverte, soit fermée. Un enfant seulement n'a pas su répondre à une question fermée et à une question ouverte. Cela nous permet d'émettre une deuxième conclusion: il ne semble pas exister de corrélation franche entre le type de question et l'incapacité d'un enfant à y répondre. Le taux d'absence de réponse se répartit assez équitablement entre les deux types de question, ce qu'illustre le tableau B où les «non-réponses» («Je sais pas») sont classées par ordre croissant d'occurrences (de r’ à I3’) ${ }^{10}$.

La lecture de ce tableau mène à une troisième conclusion: le taux d'absence de réponse à ces questions semble recouper le renvoi implicite ou explicite au terme «temps». Les questions I2' et I3' sont restées sans réponse 8 et in fois respectivement. Ce qui semble corroborer l'hypothèse de départ relative à la vacuité référentielle du terme «temps» chez les enfants de cette tranche d'âge. En revanche, les questions contenant des termes connus tels que «quand» ou «pourquoi» ont reçu davantage de réponses (I6 ou I7 pour «quand» et I4 pour «pourquoi»), alors qu'elles faisaient moins directement appel à la notion de temps. L'utilisation d'un adverbe ciblé a sans nul doute tendu à orienter l'accès à une réponse. Par conséquent, les enfants ont dans l'ensemble été plus réceptifs au type de formulation. Ce qui ne signifie pas qu'ils ont compris les questions, comme l'illustrera l'examen de leurs réponses. Si nous cherchons à présent à confronter la répartition du taux d'absence de réponse par question à l'âge des enfants, nous obtenons le tableau C.

9. Pour ces enfants, la réponse «Je ne sais pas» n'a été fournie que I ou 2 fois, voire n’a pas été fournie du tout.

10. Dans la mesure où tous les enfants ont répondu à la question "C’est quand "hier" ?", elle n'apparaît pas dans le tableau. 


\begin{tabular}{|c|c|c|}
\hline Numéro & Question & Nombre d'occurrences \\
\hline I' & Veux-tu me dessiner le temps? & I \\
\hline $2^{\prime}$ & C'est quand «demain»? & I \\
\hline 3 & C’est quand: «J'ai joué à la poupée/au ballon»? & I \\
\hline 4 & Sais-tu ce qu'est le temps? & 2 \\
\hline$s^{\prime}$ & C’est quand: «Je joue à la poupée/au ballon»? & 2 \\
\hline 6 & C’est quand: «Je jouais à la poupée/au ballon»? & 2 \\
\hline 7 & $\begin{array}{l}\text { Pourquoi je dis (la phrase) : «J'avais mangé des frites»? } \\
{[\ldots]}\end{array}$ & 4 \\
\hline 8 & Peux-tu me dire à quoi ça sert? & 5 \\
\hline 9' & Pourquoi je dis (la phrase): «Je mange des frites»? [...] & 5 \\
\hline IO' & Pourquoi je dis (la phrase): «Je mangeais des frites»? [...] & 5 \\
\hline II' & Pourquoi je dis (la phrase): «J'ai mangé des frites»? [...] & 6 \\
\hline I2' & Peux-tu me dire ce que c'est? & 8 \\
\hline $13{ }^{\prime}$ & Comment fait-on pour parler du temps? & II \\
\hline
\end{tabular}

Tableau B. Répartition des réponses «Je sais pas» fournies aux questions posées

\begin{tabular}{|c|c|}
\hline Question & Âge (réponse: «Je sais pas» - par ordre croissant) \\
\hline I & $6 \mathrm{a} 8 \mathrm{mi} 4 \mathrm{j}$ \\
\hline 2 & $6 \mathrm{a}$ \\
\hline 3 & 7armı $8 \mathrm{j}$ \\
\hline 4 & $6 \mathrm{a} 8 \mathrm{~m} 3 \mathrm{j} ; 7 \mathrm{a} 7 \mathrm{~m} 6 \mathrm{j}$ \\
\hline 5 & 6a3m26j; 7armı $8 \mathrm{j}$ \\
\hline 6 & $6 a ; 7 \operatorname{arm} 5 j$ \\
\hline 7 & $6 \mathrm{a} 3 \mathrm{~m} 26 \mathrm{j} ; 6 \mathrm{a} 8 \mathrm{~m} 3 \mathrm{j} ; 7 \mathrm{armr} 8 \mathrm{j} ; 7 \mathrm{a} 4 \mathrm{mI2j}$ \\
\hline 8 & $6 \mathrm{a} 3 \mathrm{~m} 26 \mathrm{j} ; 6 \mathrm{a} 6 \mathrm{~m} ? \mathrm{j} ; 6 \mathrm{a} 7 \mathrm{~m} \mathbf{5 j} ; 6 \mathrm{a} 8 \mathrm{~m}_{3 \mathrm{j}} ; 6 \mathrm{a} 8 \mathrm{~m}_{4} \mathrm{j}$ \\
\hline 9 & 6a; 6a7mıj; 6a8m3j; 7aзm9j; 7armı 8 ; \\
\hline Io & 6a; 6a8m3j; 7aım $5 \mathrm{j} ; 7 a ı m 6 j ; 8 a 3 m 7 j$ \\
\hline II & 6a; 6a7mıj; 6a8m3j; 6a8mı4j; 7aımı8j; 7a4mı2j \\
\hline 12 & 6a; 6а7mı 5 ; 6a8m3j; 6a8mı4j; 6a9m8j; 7a4mı2j; 7a7m6j; 7aıım6j \\
\hline $\mathrm{I} 3$ & 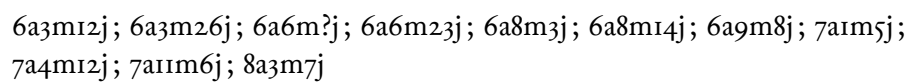 \\
\hline
\end{tabular}

Tableau C. Âge des enfants répondant «Je sais pas» aux questions posées 
Un nouveau classement de ces données par tranche d'âge pour chacun des groupes 6 et $7^{11}$ fournit les distributions suivantes (tableaux D et E) ${ }^{12}$ :

\begin{tabular}{|l|l|l|l|l|l|l|l|l|l|l|l|l|l|}
\hline Question & I & 2 & & 4 & 5 & 6 & 7 & 8 & 9 & IO & II & I2 & I3 \\
\hline «Je sais pas» & (I) & (I) & & I & I & I & 2 & $(5)$ & 3 & 2 & 4 & 5 & 7 \\
\hline
\end{tabular}

Tableau D. Répartition des réponses «Je sais pas» pour les enfants du groupe 6

\begin{tabular}{|l|l|l|l|l|l|l|l|l|l|l|l|l|l|}
\hline Question & & & 3 & 4 & 5 & 6 & 7 & & 9 & IO & II & I2 & I3 \\
\hline «Je sais pas» & & & ( $)$ & I & I & I & 2 & & 2 & 2 & 2 & 3 & 3 \\
\hline
\end{tabular}

Tableau E. Répartition des réponses «Je sais pas» pour les enfants du groupe 7

Nous notons alors la présence intéressante d'une séparation, d'une frontière, entre les questions 6 et 7 - similaire à celle observée dans le tableau B -, qui marque l'accroissement du taux d'absence de réponse à partir de la question 7 et qui correspond à la dichotomie observée entre les questions construites sur «quand» et celles construites sur «pourquoi». Par conséquent, le type de question l'emporte à nouveau sur la différence d'âge. Les enfants de la tranche d'âge de 6 ans et ceux de la tranche d'âge de 7 ans ont mieux réagi aux questions ouvertes en «quand» qu'aux questions ouvertes en «pourquoi». Partant, les enfants concernés semblent avoir fait preuve d'un début de conscience de la temporalité, celle-ci étant toutefois réduite à certains types de formulation. Ce qui pourrait indiquer que cette conscience temporelle naissante repose sur des constituants lexicaux dans ses premiers stades linguistiques. Il convient dès lors de comparer ces conclusions aux réponses fournies par les enfants.

\subsubsection{Réponses (autres que «Je sais pas»)}

Les réponses ${ }^{13}$ ont été classées par âge puisque c'est un facteur différentiel. Cela permet de proposer une synthèse des réponses à chacune des questions pour les 6 ans, les 7 ans, l'enfant de 8 ans constituant une classe. Il apparait d'emblée que les réponses des enfants de 6 ans ont été les plus nombreuses et surtout les plus pertinentes, tendance qui se remarque également chez les 7 ans où les plus jeunes d'entre eux ont formulé des réponses plus développées. Faute de place, nous proposons une synthèse des réponses obtenues ${ }^{14}$.

11. L'enfant de 8 ans n'a pu répondre à une question fermée en «pourquoi» et une question générale sur le temps.

12. Le chiffre entre parenthèses correspond au nombre total d'occurrences de réponse pour la question envisagée.

13. Le détail des réponses comme de leur analyse pourra être envoyé sur simple demande à l'auteur de l'article.

14. Les réponses ont été classées de A à $\mathrm{N}$, afin d'éviter toute confusion avec les questions auxquelles elles correspondent. 


\subsubsection{Réponses $A$ (à la question 1: «Sais-tu ce qu'est le temps?»), \\ $B$ («Peux-tu me dire ce que c'est?»), C («Peux-tu me dire à quoi ça sert?»), \\ D («Comment fait-on pour parler du temps?»)}

D'après les réponses fournies aux quatre premières questions, il est apparu que les enfants savaient selon eux ce qu'était «le temps», à l'exception de 4 enfants appartenant au groupe 6 (les plus jeunes - désormais G6), un appartenant au groupe 7 (désormais $\mathrm{G}_{7}$ ) et l'enfant du groupe 8 (désormais G8). S'ils ont donné des détails, ils ont défini «le temps» en termes d'heures, pour le G6, et en termes de temps extralinguistique, d'heures et de temps météorologique, pour le G7. À l'évidence, les termes mêmes de la question i orientent les réponses. Cependant, cette question visait précisément à cibler quelle acception de la lexie «temps» est privilégiée par l'enfant dans une question de ce type. De même, l'un des enfants du G6 n'a pas vu pas la nécessité (ou a été incapable) d'expliciter la notion et a répondu en $\mathrm{B}$ : «c'est le temps». C'est un cas intéressant que nous signalerons par la suite par la mention «6A». Les enfants du G7 ont utilisé des termes spatiaux («y aller»). L'enfant du G8 a défini le temps en termes de durée, ce qu'il a exprimé à travers l'emploi d'un verbe à l'aspect interne d'activité (i. e. non borné, atélique: «attendre»). On constate que ce qui est exprimé ressortit plus à une sensation qu'à une explication, ce qui, en faisant porter cette perception sur le verbe, illustre les conclusions de Ferreiro. L'enfant a ici traduit son expérience du «temps» par l'emploi d'un verbe dont on peut supposer que l'aspect interne lui a semblé sinon significatif, du moins apte à rendre son sentiment.

La question de l'utilité du temps a suscité l'apparition de notions chronologiques (G6). Un enfant a décrit (au présent) une succession d'actions entrecoupées d'arrêts. 6A a associé le temps à la vie. Les enfants du G6 ont donné l'impression de mieux maîtriser les notions que ceux du $\mathrm{G}_{7}$ qui ont réduit l'utilité du temps à la météorologie et à l'heure. Sur le plan essentiellement physiologique ${ }^{15}$, ces deux concepts correspondent à deux critères de taxinomie temporelle (voir, entre autres, à ce sujet: Buzsáki, 2005; Wright, 2006; Davies, 2006: 6-II ; Knierim, 2007: 42-48; Bellanger, 2008: 45-63; Rousseau, 2010: 17-39; Boroditsky, 20II : 333-34I; Buser et Debru, 20II). Le premier, le temps météorologique, traduit une perception d'ordre chronobiologique et s'inscrit dans le phénomène plus large de l'alternance jour/nuit. Le second traduit ce que l'on nomme le «temps d'horloge» ou «temps objectif» et constitue un outil créé de toutes pièces dans le but de rythmer les activités humaines (Roussel, 2009). Il est en outre intéressant de remarquer qu'un enfant du G6 a parlé du temps en termes d'espace : «le futur, c'est de l'espace» (6agm8j); d'où, pour lui, une possible unification, ou indifférenciation, des deux notions. Les réponses, conjuguées au présent, ont repris le temps utilisé dans les questions.

Le nombre de réponses proposées en $\mathrm{D}$ a été réduit. Cependant, les enfants du G6 qui ont répondu ont compris le sens global de la question et, en proposant un

15. Ce qui aura des retombées d'ordre phénoménologique. 
«outil» pour parler du temps renseignant la manière dont il pouvait être fait référence au temps, ont répondu au terme interrogatif. Leurs réponses, non construites sous forme de phrases, ont privilégié le recours aux saisons ainsi qu'à l'agenda. Le temps en question était alors pour eux un temps calendaire obéissant au retour des saisons. Les enfants du $\mathrm{G}_{7}$ ont eu plus de difficultés à s'exprimer. La seule réponse cohérente, au présent, a fait référence à un orage, c'est-à-dire au temps météorologique. On constate donc que ces deux critères de catégorisation temporelle ne sont pas aisément dissociables dans la métalangue de l'enfant.

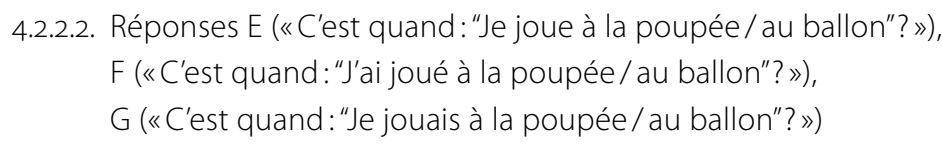

Construite sur «quand», indice temporel, la question 5 a suscité davantage de réponses (9 enfants sur II dans le G6: 8I,8I \%; 7 sur 7 dans le G7: I00 \%) conjuguées au présent à valeur générique. Les enfants du G6 ont associé ce présent à des repères quotidiens ou hebdomadaires qui reprenaient des éléments de leur expérience segmentant les activités (école, récréation, «après manger»). Le présent s'y est trouvé associé à des réitérations d'événements semblables. Un enfant a répondu en termes spatiaux ("C'est quand [...] ? - Dans la cour»), associant le lieu de l'activité avec le moment et/ou la fréquence de son occurrence. Dans ces réponses, le constituant porteur de la charge sémantique de «quand» a plutôt renvoyé à un événement («je rentre de l'école», «je joue», etc.) qu'à un moment. L'enfant $6 \mathrm{~A}$ a fourni une réponse à valeur temporelle "par défaut», dans le sens où il a spécifié une absence d'occurrence ("Quand il n'y a pas d'école»), ce qui suppose une capacité préalable à synthétiser les occurrences possibles de l'événement visé, puis à gommer la segmentation pour la commenter. Le G7 a eu une vision hebdomadaire plutôt que quotidienne des activités et a continué à unifier (ou indifférencier) le temps et l'espace. Le critère de segmentation hebdomadaire s'est donc révélé commun aux deux tranches d'âge. Il semble que l'on puisse y voir l'apparition d'une certaine notion définissable en termes de chronologie. Pour sa part, l'enfant du G8 n'a évoqué que la récurrence quotidienne.

Malgré une modification sur le plan de la conjugaison du verbe (passé composé), l'ensemble des réponses $\mathrm{F}$ à la question 6 a tendu vers la confirmation de la notion de succession et l'établissement de celle de postériorité, se construisant toujours en fonction de l'expérience des enfants. Une notion temporelle s'est trouvée associée au passé composé par une majorité d'enfants. L'amalgame temps/espace s'est poursuivi («Dans la cour»). L'utilisation du passé composé dans la question semble donc avoir sollicité le rappel d'un ou de plusieurs événement(s) antérieur(s) propre(s) à l'expérience de l'enfant concerné, dont la saillance a pour lui été prioritairement spatiale. C’est le lieu plutôt que le moment du jeu qui a dénoté le révolu.

Les réponses apportées à la question 7 (à l'imparfait) ont été conjuguées à l'imparfait, au présent, au plus-que-parfait pour le G6, et au présent et à l'imparfait pour le G7. Le nombre de réponses conjuguées a de nouveau été supérieur dans le G6, qui a utilisé l'imparfait avec une valeur d'antériorité («C'était avant»). Les réponses non 
conjuguées ont dénoté des moments dont l'antériorité semblait évidente pour les enfants concernés («Ben, lundi»; «Mercredi dernier»; «Avant que j’arrête de jouer à la poupée»). La forme temporelle s'est cette fois clairement trouvée associée sinon au temps, du moins à l'antériorité. Il en a été de même pour le G7 et le G8 dont les réponses non conjuguées ont référé au révolu. Cependant, le seul enfant qui a employé l'imparfait semble l'avoir fait en lui attribuant une valeur d'habitude (réitération d'un procès unique): «Quand je jouais au ballon?». La notion d'antériorité s'est révélée présente sur le plan de la compréhension des formes temporelles mais toujours difficile sur le plan de l'expression. Il y a encore eu référence à l'espace. Deux enfants, l'un du G6 et l'autre du G7, nous ont fait remarquer que la question avait déjà été posée. Nous pensons qu'il faut y voir le signe qu'ils n'ont pas distingué le changement de conjugaison opéré sur le verbe entre les questions 6 et 7 .

\subsubsection{Réponses H ("C'est quand "hier"? ») et I («C'est quand "demain"? »)}

Les questions 8 et 9 portaient explicitement sur l'antériorité et la postériorité, deux notions qui ont majoritairement été associées dans les réponses au phénomène de l'alternance jour/nuit chez les 6 ans comme chez les 7 ans. Il y a clairement été question du jour et du soleil par opposition au soir et à la lune, avec une mise en place de la semaine: nombreux ont été les enfants qui en ont compté les jours. L'enfant du G8 a répondu de manière explicite à cette question en proposant un raisonnement basé sur les jours de la semaine («Jeudi. Hier on était... Avant-hier on était mercredi donc je sais qu'après c'était jeudi [...]»). La notion d'antériorité, traduite par «avant», a été exprimée par quatre enfants du G6 et un du $\mathrm{G}_{7}$, et elle s'est caractérisée par son application à l'expérience des enfants. Le temps des enfants est apparu à l'échelle de leurs activités, voire «à leur échelle». Ils n'ont pas eu de vision d'ensemble. Un enfant du $\mathrm{G}_{7}$ a répondu «demain» à la question 8 , ce qui peut avoir constitué un indice de chronologie, même si celle-ci n'était à l'évidence pas encore fixée parce qu'il a répondu «hier» à la question 9, qui consistait à dater «demain». La notion est apparue plus difficile à appréhender, même si deux enfants du G6 et un du $\mathrm{G}_{7}$ ont répondu en utilisant le futur simple. Les autres réponses conjuguées l'ont été au présent. Dans l'ensemble, la lexie «demain» comme la lexie «hier» ont plus volontiers déclenché des réponses conjuguées correctement que les formes verbales proprement dites. C'est à nouveau l'alternance jour/nuit, donc la succession, qui a été proposée comme mode d'illustration de la référence du terme «demain», avec insistance sur la nuit - y compris pour l'enfant du G8 -, alternance accompagnée d'un renvoi possible à l'espace («en passant par la nuit»).

\subsubsection{Réponse J («Pourquoi je dis (la phrase) : "Je mange des frites"? (peut-on dire autre chose?)»)}

La question Io, construite sur "pourquoi», visait plus spécifiquement une analyse du temps sur le verbe $«$ manger ${ }^{16}$ (au présent). La majorité des enfants des deux

16. Lors de la passation, il y a eu insistance prosodique sur «je mange des frites». 
groupes a répondu en termes de cause, avec des réponses débutant par "parce que». Ils n'ont donc pas/plus réagi au temps porté par le verbe, même s'ils ont utilisé le présent dans leurs réponses. Nous pouvons donc conclure, au vu de leurs réponses aux questions 5, 6 et 7, qu'ils semblent avoir davantage prêté attention au temps du verbe lorsque celui-ci était sous-tendu par une référence temporelle telle que «quand». Partant, nous pouvons déduire qu'ils semblent avoir été plus réceptifs au lexique temporel qu'aux conjugaisons, bien qu'ils aient su en employer plusieurs. Ce qui signifie qu'ils n'ont auparavant pas semblé associer un sémantisme particulier aux formes conjuguées. Un seul enfant, parmi les plus jeunes (G6), a répondu en ciblant la forme verbale («Je pense que c'est parce que c'est le midi»). De manière étonnante, $6 \mathrm{~A}$ n'a pas su répondre à cette question.

\subsubsection{Réponse K («Pourquoi je dis (la phrase) : "J'ai mangé des frites"? [...]»)}

Le présent cédait la place au passé composé dans cette question. Les réponses, bien que portant prioritairement sur la cause, ont mélangé le présent et le passé composé (G6), auxquels le G7 et le G8 ont ajouté l'imparfait. Ce qui semble infirmer la conclusion précédente: cette fois-ci, les enfants ont réagi à la forme temporelle. Cependant, leurs réponses ont été moins justes et précises que dans les cas précédents. Sur les trois emplois du passé composé parmi les enfants du G6, deux étaient résultatifs, donc aspectuels, et un combinait temps et aspect («Après qu'on a mangé»), semblant ainsi d'abord insister sur la postériorité («après») puis sur le résultat («on a mangé»). Un enfant du même groupe a employé le futur proche («[...] on sait qu'on va manger des frites»). À nouveau, les situations évoquées s'inscrivaient dans un cadre personnel (menu, cantine, courses, faim), quelle qu'ait été la tranche d'âge concernée.

\subsubsection{Réponse L («Pourquoi je dis (la phrase): "Je mangeais des frites"? [...]»)}

La question I2 se caractérisait par un passage à l'imparfait associé à l'utilisation de l'adverbe "pourquoi». Les enfants du G6 ont massivement recouru à la notion d'antériorité en incluant le renvoi à «hier» (6A) ou à un moment révolu qui, toutefois, appartenait à leur expérience personnelle, tout en répondant à la cause ( 5 réponses sur 8 ont débuté par «Parce que...»). Les conjugaisons utilisées ont été le présent, le passé composé ou l'imparfait, ce dernier étant prioritairement réservé à l'expression de la cause.

\subsubsection{Réponse M («Pourquoi je dis (la phrase): "J'avais mangé des frites"? [...] »)}

La question I3 était intéressante parce qu'elle était construite sur le plus-que-parfait, étudié en cours moyen $2^{\mathrm{e}}$ année $\left(\mathrm{CM}_{2}\right)$. Et pourtant, deux enfants du G6 l'ont utilisé dans leurs réponses, l'un cherchant à répondre à la cause avec des éléments temporels («Parce que hier/mardi j'avais marché»). 6A a exprimé clairement la double antériorité, ce qui rendait compte de sa capacité à répondre correctement aux questions qui avaient porté sur l'antériorité simple. Sa maitrise des phénomènes et la clarté de son expression étaient étonnantes pour un enfant de cet âge 
(Sabeau-Jouannet, 1977: 199). Par comparaison, aucun des enfants du G7 ou du G8 n'a utilisé le plus-que-parfait (Vandenplas-Holper, 1975; Fayol, 1982; Labelle, 1994: 99; Roussel, 2007b). Un autre enfant du G6 a proposé une réponse singulière: à l'imparfait (pour une question au plus-que-parfait), seule la conjugaison du verbe étant modifiée par rapport à la réponse au présent qu'il avait fournie à la question I2 (conjuguée à l'imparfait): «Parce que j’avais faim» (I3) // «Parce que tu as faim» (I2). Cet enfant semble ainsi avoir réagi en termes de sphères temporelles et a peut-être tenté d'exprimer la temporalité sur l'auxiliaire plutôt que sur le verbe (Cotte, 1987: IO2 ${ }^{17}$ ), ou a dissocié les formes en accord avec l'opposition question/réponse (ou rien de tout cela). Un enfant du G7 a semblé exprimer une «double postériorité»: «[...] on a envie de les manger mais après on n'a pas envie de les manger», toujours en réponse à la cause («Parce que $[\ldots] »)$.

\subsubsection{Réponse N («Veux-tu me dessiner le temps?»)}

Certains enfants ont explicité leur accord pour dessiner le temps. Un enfant du G6 a fait référence à la chronologie tout en ne sachant pas la nommer. Toutefois, il l'a associée aux phénomènes météorologiques. De même, si des enfants du G7 ont commenté leur accord, ils l'ont exprimé en termes météorologiques, en faisant notamment appel à la pluie et au soleil. Ces commentaires ont tous été effectués au présent.

La seconde étape de l'étude consiste à s'intéresser plus particulièrement aux procédés linguistiques mis en œuvre dans ces réponses, notamment le choix et l'utilisation des verbes.

\section{Analyse linguistique des réponses}

La répartition et le type de verbes utilisés par les enfants dans leurs réponses ont été analysés en fonction des critères avancés d'ordinaire (et présentés plus haut) dans l'étude des propriétés aspectuo-temporelles du langage utilisé par les jeunes enfants en lien avec le renvoi au révolu: présence ou absence d'un résultat observable aux verbes employés, type de verbes employés (Andersen et Shirai, 1995), répartition de l'imparfait et du passé composé en fonction de l'aspect interne des verbes (Andersen, 1993; Howard, 2002), sémantisme des lexèmes verbaux (Müller, 1974). Un total de 253 verbes a été utilisé dans les I3 ou I4 réponses fournies par chacun des i8 enfants interrogés. La répartition du nombre de verbes pour chaque tranche d'âge par rapport au total des verbes est la suivante:

17. «[...] les enfants acquièrent tardivement (le passé composé temporel). On peut penser que [...] l'auxiliaire doit alors se charger d'assurer le repérage temporel et prend de ce fait l'imparfait, qui est le seul temps du passé disponible pour les jeunes enfants.» 


\begin{tabular}{|l|l|l|l|}
\hline Âge & Nombre d'enfants & Verbes & Pourcentage \\
\hline 6 & IO & I28 & 5 I $\%$ \\
\hline 7 & 7 & II2 & $44 \%$ \\
\hline 8 & I & I3 & $5 \%$ \\
\hline
\end{tabular}

Tableau F. Répartition des verbes par tranche d'âge

\subsection{Résultat observable}

La recherche de présence d'un résultat observable (correspondant traditionnellement à un aspect grammatical) - test de Bronckart (selon Andersen et Shirai, 1995: 745) -, n'est pas fondamentalement significative puisqu'elle donne les résultats suivants:

\begin{tabular}{|l|l|l|l|}
\hline Âge & Nombre d'enfants & Résultat & Pourcentage \\
\hline 6 & IO & I3 & $5 \%$ \\
\hline 7 & 7 & 6 & $2 \%$ \\
\hline 8 & I & 0 & $0 \%$ \\
\hline
\end{tabular}

Tableau G. Répartition d'un résultat observable par tranche d'âge

Cependant, on peut se demander si ce critère de résultativité est bien ce qui est en jeu dans de tels énoncés, d'où le faible pourcentage observé, et s'il ne faudrait pas plutôt envisager une autre relation au présent qui ne serait ni aspectuelle (résultative), ni temporelle, mais d'une autre nature, qui unifierait les deux paramètres en une seule perception (Cotte, 1987: 105) dont l'enfant ne retiendrait que ce qui importe pour lui : le schéma sémantique du procès dénoté par le verbe. Ce ne serait pas tant l'après du procès ou son rapport au présent de l'énonciation que sa nature même qui importerait pour l'enfant, tout simplement, puisqu'à cet âge il est incapable, sur le plan physiologique, d'avoir le recul nécessaire pour construire la décentration que sous-tendent les interprétations aspectuelle (grammaticale) et temporelle. Dans de telles occurrences, c'est l'adulte, capable de décentration, qui assigne un résultat au verbe employé, mais qu'en est-il vraiment de l'enfant? Il semble donc plus cohérent de focaliser l'analyse sur l'aspect interne des lexèmes verbaux.

\subsection{Verbes}

L'étude de l'aspect lexical est ainsi plus intéressante. Globalement, on relève une majorité de verbes d'état ( 95 sur $253=38 \%$ ) et d'activité ( 82 sur $253=32 \%$ ). Viennent ensuite les accomplissements (4I sur 253 = I6 \%) puis les achèvements (19 sur $253=8 \%$ ). Par type de verbes, toutes conjugaisons confondues dans un premier temps, la répartition selon les groupes d'âge est comme suit: 
- État (type «être»: 95 verbes $-38 \%)$ :

\begin{tabular}{|l|l|l|l|}
\hline Âge & Nombre d'enfants & État & Pourcentage \\
\hline 6 & IO & $\begin{array}{l}\text { 49 sur I28 verbes en } \\
\text { tout pour le G6 }\end{array}$ & $38 \%$ \\
\hline 7 & 7 & 39 sur II2 verbes & $35 \%$ \\
\hline 8 & I & 7 sur I3 verbes & $54 \%$ \\
\hline
\end{tabular}

Tableau H. Répartition des verbes d'état par tranche d'âge

- Activité (type «lire»: 82 verbes $-32 \%$ )

\begin{tabular}{|l|l|l|l|}
\hline Âge & Nombre d'enfants & Activité & Pourcentage \\
\hline 6 & IO & 37 sur I28 verbes & $29 \%$ \\
\hline 7 & 7 & 40 sur II2 verbes & $36 \%$ \\
\hline 8 & I & 5 sur I3 verbes & $38 \%$ \\
\hline
\end{tabular}

Tableau I. Répartition des verbes d'activité par tranche d'âge

- Accomplissement (type «lire un article»: 4I verbes - I6 \%)

\begin{tabular}{|l|l|l|l|}
\hline Âge & Nombre d'enfants & Accomplissement & Pourcentage \\
\hline 6 & IO & 20 sur I28 verbes & I6 \% \\
\hline 7 & 7 & 2I sur II2 verbes & 19 $\%$ \\
\hline 8 & I & O sur I3 verbes & O $\%$ \\
\hline
\end{tabular}

Tableau J. Répartition des verbes d'accomplissement par tranche d'âge

- Achèvement (type «partir»: 19 verbes - $8 \%$ )

\begin{tabular}{|l|l|l|l|}
\hline Âge & Nombre d'enfants & Achèvement & Pourcentage \\
\hline 6 & IO & I5 sur I28 verbes & I2 $\%$ \\
\hline 7 & 7 & 4 sur II2 verbes & $4 \%$ \\
\hline 8 & I & O sur I3 verbes & $0 \%$ \\
\hline
\end{tabular}

Tableau K. Répartition des verbes d'achèvement par tranche d'âge 
On remarque que l'on est plus dans le descriptif que dans le narratif, c'est-à-dire que la proportion de suites événementielles est moindre. On est également davantage dans le témoignage, ce qui correspond à une "vision» interne des procès (pour reprendre les termes de Labelle, 2002): l'enfant semble (re)vivre l'épisode qu'il mentionne (y compris lorsqu'il emploie un présent à valeur spécifique ou générique).

Cet emploi des verbes est logique si on le rapporte au stade du développement physiologique, notamment mémoriel, des enfants concernés: le flux temporel se met en place progressivement via la mémoire sémantique ${ }^{18}$ (et l'augmentation du lexique, y compris verbal, à la disposition de l'enfant). En revanche, la faculté de «décentration» piagétienne qui permet le recul, le «regard extérieur» sur les événements, et signe l'acquisition de la notion de temps, n'est pas encore en place dans la mémoire épisodique ${ }^{19}$. C'est ce qu'indique l'incapacité, ou la difficulté, des enfants à agencer les événements selon une chronologie objective (voir 5.I). Il se peut que l'emploi de verbes d'accomplissement et d'achèvement soit également en corrélation avec les compétences lexicales, au sens large, de l'enfant. On note ainsi que l'enfant du G8 utilise bien moins de verbes d'état et d'activité que ceux du G6 et du $\mathrm{G}_{7}$, et aucun verbe d'accomplissement et d'achèvement. Ceci pourrait indiquer le caractère prioritairement individuel du développement et de l'acquisition de la notion de temps ainsi que de sa métalangue, susceptibles d'être plus ou moins longs selon les enfants. Les chiffres linguistiques soulignent la tendance observée sur le plan extralinguistique: tout comme l'enfant du G8 a semblé opérer une entrée plus tardive que ceux du G6 et du G7 dans sa temporalité - ce qu'indiqueraient ses références pragmatiques et phénoménologiques -, on relève que sa maitrise lexicale est, de même, plus pauvre. Par conséquent, compétences linguistique et métalinguistique semblent liées pour ce qui est du temps, dans la mesure où il est nécessaire que les enfants disposent d'un vocabulaire adapté pour être capables de traduire les phases d'appropriation de la notion temporelle. Toutefois, la mise en place de ce vocabulaire n'est pas acquise d'emblée; elle a sa propre temporalité, qui se définit par son caractère progressif. En outre, le dessin de l'enfant du G8 semble indiquer que la métalangue n'est possible que lorsque certaines étapes ont été franchies ${ }^{20}$. C'est ainsi que la notion de flux temporel ne semble pouvoir être envisagée que lorsque l'alternance jour/nuit devient significative et est énoncée en

18. La mémoire sémantique relève de la mémoire déclarative (ou mémoire à long terme), mémoire qui se développe avec l'âge, et favorise l'acquisition, la représentation et l'utilisation des connaissances relatives à des faits et des événements. Cette connaissance est explicite (Ullman, 2006: 263), c'est-à-dire qu'elle est disponible à la conscience.

19. Autre sous-mémoire déclarative, elle traite des événements autobiographiques dans lesquels les dates, les lieux et les émotions liés à ces événements jouent un rôle primordial (Desmarais et al., 1997).

20. Le dessin de l'enfant du G8 s'est concentré sur la représentation d'activités rythmées par l'alternance jour/nuit, un enfant étant présent dans la scène, ce qui suggère une référence à son expérience personnelle; l'enfant a notamment dessiné une table dressée pour le repas, comme l'ont fait deux des enfants du G6. Sans doute cela a-t-il schématisé son entrée, à son rythme, dans la temporalité, à travers la mise en place de la succession. C'est du reste ce qu'a indiqué son commentaire au fur et à mesure que le dessin prenait forme: «Je fais un lit pour montrer que c'est hier». 
tant que telle par l'enfant ${ }^{21}$. Les compétences métalinguistiques témoigneraient ainsi plus de l'avancement du développement cognitif de l'enfant que de l'état de ses compétences linguistiques.

Le rapport entre état et activité est différent selon les groupes d'âge. Le taux des activités diminue dans le G6: 37 (29\%) contre 49 (38\%); comme dans le G8: 5 (38\%) contre $7(54 \%)$; tandis qu'il augmente très légèrement dans le $\mathrm{G}_{7}: 40(36 \%)$ contre 39 $(35 \%)$. Les résultats semblent indiquer que l'évolution état/activité est inversée d'un groupe d'âge à l'autre comme à l'intérieur d'un même groupe d'âge. Chez les 6 ans, l'état est prédominant. Tant qu'il en est ainsi, les activités sont en nombre moins élevé. À l'inverse, les activités dépassent légèrement les états chez les 7 ans. Ceci tend à indiquer que le développement de l'emploi des activités devient possible à partir d'une certaine maitrise de l'état, ou que les deux coexistent et que leur répartition évolue au fur et à mesure que l'enfant grandit, ce qui du reste serait parfaitement compatible avec le sémantisme de ces verbes. Il est vrai que puisque le nombre d'enfants de 6 ans est supérieur à celui des enfants de 7 ans (Io contre 7), il est tout à fait possible que les pourcentages reflètent cette disparité. Cependant, si tel est le cas, elle ne semble plus jouer pour les accomplissements où les io enfants du G6 produisent 20 occurrences et les 7 enfants du $\mathrm{G}_{7}$ en produisent 2I. Si l'effectif est un facteur influant, alors dans le cas des accomplissements, cela signifierait bien que les enfants du G6 en produisent moins que ceux du G7, puisqu'à Io ils rassemblent 20 occurrences tandis que les enfants du G7 en rassemblent 2i à 7. Toutefois, que penser alors de la répartition des occurrences des achèvements, qui est de is pour le G6 contre 4 pour le G7? Dans ce cas, même si les enfants du G6 sont plus nombreux, ils semblent bel et bien posséder un éventail de types de verbes plus large que les enfants du G7.

Il reste que pour le groupe observé, la gestion des accomplissements et des achèvements semble naissante comparativement à celle des états et des activités, et seconde dans le processus d'acquisition et/ou d'emploi des types de verbes. L'emploi de ce type de verbes serait donc corrélé au stade de développement des capacités de décentration sur le plan physiologique.

L'examen détaillé de la catégorie de l'état indique une prédominance de l'emploi du verbe «être» par rapport à «avoir», hormis pour l'enfant du G8. Au sein de la catégorie de l'état, le G6 emploie 37 fois le verbe «être» (47\%) et 4 fois le verbe «avoir» (8\%) sur un total de 49 verbes d'état (soit $29 \%$ contre $3 \%$ sur un total de i2 8 verbes tous types confondus). Le G7 emploie I9 fois le verbe «être» (49\%) et I4 fois le verbe «avoir» $(36 \%)$ sur un total de 39 verbes d'état (soit i7 \% contre I3 \% sur un total de II2 verbes tous types confondus). Quant à l'emploi que fait le G8 des verbes d'état, il est équilibré: 4 fois «être» et 3 fois «avoir» sur un total de 7 verbes d'état. L'enfant de ce groupe ne disposait donc que de ces deux verbes dans la catégorie d'état, tandis que

21. 6agm8j: «Demain. Demain ce sera [...] ben il faut que la nuit passe entièrement»; 6azmız: «C'est le jour qui sera après en passant par la nuit»; 7a4mı $\mathrm{j}$ : «Après ce soir, c'est quand on dort»; 8a: «Samedi. Il faut dormir». 
les autres enfants ont utilisé d'autres verbes d'état. D'après ces données, et sans doute sans grande surprise, les deux premiers verbes d'état, sinon à être acquis, du moins à être utilisés le plus volontiers, sont «être» et «avoir», respectivement. Parmi ceux-ci, les enfants du G6 ont massivement employé «être» par rapport à «avoir», tandis que ceux du $\mathrm{G}_{7}$ ont réduit la différence entre ces deux verbes à 5 occurrences (elle se monte à 33 occurrences chez les 6 ans); quoi qu'il en soit, «être» reste majoritaire, y compris chez l'enfant du G8 (4 occurrences contre 3 pour «avoir»).

L'inventaire des verbes utilisés, d'après Howard (2002), a consisté à chercher à savoir si ces verbes correspondaient aux 22 verbes les plus fréquents du français mis en évidence par Müller (1974) ${ }^{22}$. L'opération s'est révélée plutôt significative, même si les questions ciblées sont construites sur certains verbes (dont seuls 4 font partie de ladite liste: «être», «savoir», «parler» et «dire») que les enfants ont été incités à reprendre dans leurs réponses. En effet, l'ordre dans lequel les verbes de la liste apparaissent est établi en fonction de leur fréquence d'emploi dans un corpus oral (Gougenheim et al., 1964). Selon ce critère, on remarque que les ir premiers (de «être» à «venir») ont bien été utilisés par les enfants, puis ensuite plus rien hormis «passer» (II fois : 7 [G6] et 4 [G7]), «parler» (I fois: G6) et «aimer» (4 fois : $3[\mathrm{G} 6]$ et $\left.\mathrm{I}\left[\mathrm{G}_{7}\right]\right)$. «Aimer» est indirectement induit par la question construite sur la locution «manger des frites», son utilisation n'est donc pas libre de tout contexte. L'emploi de "passer» est plus intéressant dans la mesure où il apparaît dans des «blocs syntactico-sémantiques»: «ça passe», «le temps qui passe» (3 occurrences), "ça sert à passer le temps», «je passe du temps», «on passe de mercredi à jeudi», «en passant par la nuit», «le jour qui passe», «chaque jour qui passe»... Ces blocs semblent répondre aux critères de Tomasello (2000: 66 et 76 ) lorsqu'il décrit l'emploi des premières combinaisons lexicales et le caractère significatif de leur récurrence ramenée à leur fréquence dans la vie quotidienne. Ces constructions types, qui sur le plan mémoriel relèvent à la fois des mémoires sémantique et épisodique, forment selon Tomasello un inventaire qui définit les compétences langagières des enfants. En ce qui nous concerne, nous constatons que c'est à travers l'emploi récurrent de ces blocs que la notion de flux temporel est exprimée. Le verbe utilisé, "passer» conjugué au présent, est l'un des verbes d'activité relevés dont le sémantisme véhicule précisément la notion de mouvement continu, en parfaite affinité avec les propriétés durative et atélique des verbes d'activité. Le verbe nomme clairement son signifié. La question qui se pose est alors de savoir si le verbe est utilisé pour son sémantisme propre ou pour sa combinaison avec le temps telle qu'elle est intériorisée dans les habitudes de communication de l'enfant. Toujours est-il que la notion temporelle est prioritairement traduite sur le plan lexical et non par la conjugaison. Un lexique qui rejoue l'expérience vécue et perçue.

22. Verbes dont voici la liste : «être», «avoir», «faire», «dire», «aller», «voir», «savoir», «pouvoir», «falloir», «vouloir», «venir», «prendre», «arriver», «croire», «mettre», «passer», «devoir», "parler», «trouver», «donner», «tenir» et «aimer». On comparera avec intérêt ces données à celles concernant le français L2 (Noyau, 2005 et 2008). 
Pour ce qui est plus précisément de l'emploi de l'imparfait et du passé composé ainsi que de leur répartition par types de verbes, l'enquête est globalement conforme aux critères d'Andersen (1993), appliqués aux apprenants du français langue seconde par Howard (2002: 189). C'est-à-dire que les états et les activités sont bien conjugués à l'imparfait, les achèvements et les accomplissements étant, pour leur part, conjugués au passé composé (Cotte, 1987: 134). Il serait à juste titre légitime d'envisager que cette répartition puisse être guidée par le temps employé dans la question qui flouterait les coordonnées spatiotemporelles de la situation d'énonciation. Il semble par conséquent difficile d'apporter une explication claire aux motivations réelles de l'emploi de ces flexions temporelles. En revanche, on note que cette répartition s'opère même lorsque le verbe de la question est conjugué à un autre temps que celui utilisé par les enfants dans la réponse (exemple: question i2 à l'imparfait, réponse $\mathrm{L}$ au passé composé). Le temps utilisé dans la question n'est alors plus un facteur contestable. Les enfants semblent avant tout conjuguer leurs verbes en fonction de leur sémantisme interne. La répartition s'effectue également lorsque les enfants cherchent à expliciter un lexème de la question (exemple: question 8 contenant «hier», réponse $\mathrm{H}$ conjuguée à l'imparfait). Ce point est très intéressant dans la mesure où il apparaît que ces explications, lorsqu'elles sont conjuguées à l'imparfait, font intervenir des notions relevant d'expériences personnelles (sujets à la $\mathrm{I}^{\mathrm{re}}$ personne du singulier, arguments liés à la condition de l'être humain dans son environnement immédiat [«avoir faim», «être affamé», «faire beau», «être + date» - «hier»- ou mention du temps météorologique]). Les explications fournies au passé composé relèvent à l'inverse du champ général ou indéfini (sujets génériques [«on», «tout le monde»], procès mentionnés certes vécus par les enfants mais liés à des événements ayant eu lieu en collectivité [cantine, récréation, etc.]). Il apparait enfin que les questions à l'imparfait reçoivent plus de réponses conjuguées correctement que les questions au passé composé (exemple: question 6 au passé composé, réponses F à l'imparfait, au présent ou au futur proche, ou emploi d'un lexique dédié [«venir de», «avant», «après»]). Partant, il semblerait que la nature interne du lexème verbal (état, activité) motive l'emploi de l'imparfait.

Globalement, on observe donc une prédominance de formes verbales qui se distinguent par des propriétés aspectuelles internes récurrentes. Néanmoins, une fois conjuguées, ces formes ne revêtent pas véritablement une fonction temporelle au sens grammatical du terme. Elles reflètent en priorité la mise en place de compétences linguistiques tournées vers l'expression d'une temporalité extralinguistique, voire pragmatique, basée sur des perceptions sensorielles et des propriétés mémorielles. En outre, ces formes verbales sont moins nombreuses que d'autres procédés linguistiques, notamment lexicaux, qui visent également à dénoter le temps extralinguistique.

\section{Discussion}

Cette recherche avait pour but d'illustrer une étude transdisciplinaire de la temporalité qui portait sur le statut du temps et de l'aspect et a mis en évidence le rôle certain 
de facteurs biocognitifs dans la structuration spatiotemporelle de l'être humain, ainsi que la manière dont celui-ci exprime cette structuration dans les langues naturelles.

Parmi ces facteurs se trouve la perception visuelle (de la présence ou de l'absence) de la lumière, qui constitue l'un des premiers indices temporels du "point de vue» cérébral. Étant donné que la linguistique attribue les premiers indices grammaticaux de la temporalité à l'aspect et au temps (Klein, 1994; Dietrich et al., 1995), il nous a semblé pertinent de chercher à savoir si ces catégories grammaticales reposaient de quelque manière que ce soit sur la phénoménologie temporelle ${ }^{23}$, voire, dans quel sens s'effectuait l'incidence, si incidence il y avait (Paradis et Crago, 2000: 84I), pour éventuellement, à terme, envisager une redéfinition de la catégorie de l'aspect comme du «point de vue» que cette catégorie est censée traduire.

En tant que biolinguiste, nous nous sommes concentrée sur les productions des enfants concernés, sans pour autant occulter les données cognitives récentes relatives à la conception du temps chez l'être humain (Bellanger, 2008: 45-63; Boroditsky, 20II: 333-34I; Buzsáki, 2005; Damazio, 2006: 34-4I ; Davies, 2006: 6-II; Droit-Volet, 2000 et 20II ; Droit-Volet et al., 2006; Izard et al., 201I : 319-332; Desmarais et al., 1997; Knierim, 2007: 42-48; Kraft, 2007: 62-65; Planche, 1998, 1999 et 2000; Spelke, 20II : 287-317; Sweetser et Nunez, 2006: 40I-450; Ullman, 2006: 235-273; Vincent, 2007; Wright, 2006), notamment le lien fondamental lumière/perception visuelle/noyau suprachiasmatique ${ }^{24}$ (Vincent, 2007; Roussel, 2009) ainsi que la planification propre à l'hémisphère gauche, y compris chez le nourrisson et l'enfant en âge préscolaire.

Au terme de la synthèse des réponses proposées par les enfants, il apparait que la proportion de réponses d'ordre aspectuel ne gomme pas les spécificités des réponses dénotant une chronologie, une antériorité ou une succession (de procès, de jours, etc.). Les notions de temps, lorsqu'elles ont existé, ont davantage été assignées au lexique qu'aux marques de conjugaison en propre. Ce lexique se caractérise par un renvoi à des événements relatifs à l'expérience personnelle des enfants, comme c'est le cas pour certains emplois aspectuels. Ce qui d'une part, montre une importance certaine de ce facteur personnel dans la construction des compétences liées à la temporalité, et d'autre part, laisse entière la question de la compréhension, par les enfants, de ces formes temporelles en tant qu'indices temporels, tout au moins en ce qui concerne les enfants observés. Nous avons cependant relevé quelques emplois cohérents de ces formes en contexte, notamment pour ce qui a été du passé composé et de l'imparfait. Par ailleurs, la référence à l'espace, quoique rare proportionnellement à l'ensemble des réponses fournies, a été constante, ce qui

23. Smith et Weist (1987: 388) posent que les quatre aspects lexicaux de Z. Vendler seraient des variantes d'un universel cognitif.

24. Petite structure abritant ce que l'on nomme «l'horloge biologique» de l'être humain, qui se situe à la base de l'hypothalamus et a la particularité de maintenir une activité rythmique constante. Les neurones de ce noyau sont étroitement liés à la rétine. 
plaide en faveur d'une prise en compte des éléments spatiaux, y compris langagiers, dans la conceptualisation de la temporalité.

Les données ont essentiellement permis de démontrer les difficultés de la primauté de l'aspect, ainsi que la non-synchronisation des maîtrises du temps extralinguistique et linguistique. Les enfants observés ont en effet, le plus souvent à propos, utilisé des formes temporelles dont ils n'étaient pas encore censés maîtriser la valeur temporelle. Ces enfants n'ont pas plus semblé percevoir la conjugaison comme un indice temporel en propre, que délibérément lui assigner un rôle particulier. Ils ont néanmoins été capables d'employer plusieurs formes temporelles: le présent, le passé composé, l'imparfait, jusqu'au plus-que-parfait.

La question des relations temporelles est décidément très complexe et implique divers paramètres qui, aux âges concernés, semblent l'avoir emporté sur le temps linguistique. En effet, le temps des enfants s'est révélé capable d'être cyclique et non uniquement rectiligne. Ses schématisations ont également indiqué qu'il pouvait psychiquement être représenté comme une entité mouvante. Le temps en question s'est prioritairement construit sur des perceptions visuelles d'alternances lumineuses ${ }^{25}$ (ce qui n'apparaît pas dans Droit-Volet, 200I : 36) puis des successions, sur lesquelles sont venues se greffer les notions (i) de postériorité puis (ii) d'antériorité, dans cet ordre. Ces perceptions peuvent dès lors être considérées comme définitoires du processus d'élaboration du temps extralinguistique, lequel apparaît comme une nécessité physiologique et phénoménologique préalable au développement de toute conscience temporelle sur le plan linguistique ${ }^{26}$.

De fait, les temps grammaticaux n'ont guère suscité de réaction, hormis chez quelques rares (très jeunes) enfants qui ont unifié temps et espace. L'ensemble des enfants a davantage réagi au lexique qui a été compris en termes de temps météorologique ou d'alternance entre le jour et la nuit ${ }^{27}$ ramenés à leurs expériences personnelles.

\section{Conclusion}

La fonction temporelle des flexions verbales n'a pas été démontrée, du moins pour les enfants de l'enquête qui ont semblé être davantage concernés par la construction de leur propre temporalité, en accord avec leurs propriétés physiologiques, notamment perceptives, mémorielles et émotionnelles. L'emploi cohérent de formes temporelles

25. Perceptions qui participent des relations primitives de l'être humain au monde réel.

26. Le phénomène a été relevé chez les apprenants polophones du français langue seconde (PaprockaPiotrowska, 2002: 232)

27. Le rôle de ce phénomène naturel a été évoqué dans l'analyse linguistique du français comme de l'anglais: voir Benveniste (I980 [1974] : 7I), Cotte (I99I : 132-I33), Gosselin (1996: I54) et O’Kelly (1994:33). Nous espérons avoir souligné son caractère primordial dans l'élaboration de la grammaire du temps, qu'il semble manifestement délicat d'envisager indépendamment de toute considération biocognitive. 
masque ainsi des divergences conceptuelles de l'arrière-plan extralinguistique sur lequel elles s'élaborent. En outre, se pose la question de l'uniformité de la compétence métalinguistique - qui semblait être un préalable stable à l'étude -, dans la mesure où certains des enfants observés (notamment $6 \mathrm{~A}$ ou l'enfant «6a7mij») ont fait preuve de compétences particulières quant à la conception du temps.

Autant d'observations qui ne sauraient qu'encourager des prolongements sur cette délicate question aspectuo-temporelle, notamment en étudiant les compétences d'enfants qui présentent des dispositions particulières (Planche, 1998, 1999 et 2000; Roussel, 2007b; Corbin et al., 2OI2a; Siaud-Facchin, 20I2) ou de faibles performances (Corbin et al., 2012b).

\section{Bibliographie}

ANDERSEN, R. 1986. El desarollo de la morfología verbal en el espaňol como segundo idioma. In J.M. MeIsel (éd.), Adquisición del lenguaje - Aquisição da linguagem. Francfort-surle-Main : Vervuert Verlag: II5-I38.

Andersen, R. 1990. Models, Processes, Principles and Strategies: Second Language Acquisition Inside and Outside the Classroom. In B. Van Patten et J.F. Lee (éd.), Second Language Acquisition - Foreign Language Learning. Clevedon : Multilingual Matters : $45^{-78}$.

Andersen, R. 1993. Four Operating Principles and Input Distribution as Explanations for Underdeveloped and Mature Morphological Systems. In K. Hyltenstam et Å. Viberg (éd.), Progression and Regression in Language. Cambridge : Cambridge University Press : 309-339.

Andersen, R. et Shirai, Y. 1994. Discourse Motivations for some Cognitive Acquisition Principles. Studies in Second Language Acquisition I6 (2): 133-156.

Andersen, R. et Shirai, Y. i995. The Acquisition of Tense-Aspect Morphology: A Prototype Account. Language 7I (4): 743-762.

Andersen, R. et Shirai, Y. 1996. The Primacy of Aspect in First and Second Language Acquisition: The Pidgin-Creole Connection. In W.C. Ritchie et T.K. Bhatia (éd.), Handbook of Second Language Acquisition. San Diego - New York - Boston : Academic Press : 527-570.

Bellanger, B. 2008. Quelle est la nature du temps? Science et vie: Les io plus grandes énigmes de la science I091 : 45-63.

Benveniste, É. 1976 [1966]. Problèmes de linguistique générale. Tel. Paris: Gallimard. T. I. Benveniste, É. I980 [1974]. Problèmes de linguistique générale. Tel. Paris: Gallimard. T. II. Berko, J. 1958. The Child's Learning of English Morphology. Word I4: I50-I77.

Bickerton, D. 1974. Creolization, Linguistic Universals, Natural Semantax and the Brain. University of Hawaii Working Papers in Linguistics 6 (3): I24-I4I.

Bickerton, D. 198I. Roots of Language. Ann Arbor: Karoma.

Bickerton, D. 1984. The Language Bioprogram Hypothesis. Behavioral and Brain Sciences 7 (2): $173-188$. 
Bishop, D.V.M. 1994. Grammatical Errors in Specific Language Impairment: Competence or Performance Limitations? Applied Psycholinguistics 15: 507-550.

Bloom, L., Lifter, K. et Hafitz, J. i98o. Semantics of Verbs and the Development of Verb Inflection in Child Language. Language 56 (2):386-4I2.

Boroditsky, L. 20II. How Languages Construct Time. In S. Dehaene et E.M. Brannon (éd.), Space, Time and Number in the Brain. Searching for the Foundations of Mathematical Thought. San Diego: Elsevier - Academic Press: 333-34I.

BroncKart, J.-P. 1976. Les modes d'expression de l'aspect dans le langage de l'enfant. Bruxelles: Dessart et Mardaga.

Buser, P. et Debru, C. 2oIr. Le temps, instant et durée. De la philosophie aux neurosciences. Paris: O. Jacob.

BuzsákI, G. 2005. Neuroscience: Neurons and Navigation. Nature 436 (7052) : 78I-782.

BybeE, J. I99i. Natural Morphology: The Organization of Paradigms and Language Acquisition. In T. Huebner et C.A. Ferguson (éd.), Crosscurrents in Second Language Acquisition and Linguistic Theories. Amsterdam - Philadelphie: J. Benjamins: 67-92.

Camos, V., Mora, G. et Oberauer, K. 2oio. Adaptive Choice in Strategies of Maintenance in Verbal Working Memory. SIst Annual Meeting of the Psychonomic Society. Saint-Louis (Missouri).

Chomsky, N. 1993. A Minimalist Program for Linguistic Theory. In K.L. Hale et S.J. Keyser (éd.), The View from Building 20: Essays in Linguistics in Honor of Sylvain Bromberger. Cambridge: MIT Press : I- 52 .

Clahsen, H., Penke, M. et Parodi, T. 1993. Functional Categories in Early Child German. Language Acquisition 3 (4): 395-429.

Clark, E.V. 1985. The Acquisition of Romance, with Special Reference to French. In D.I. Slobin (éd.), The Crosslinguistic Study of Language Acquisition. Hillsdale - Londres: L. Erlbaum. Vol. I, The Data: 687-782.

Coppens, Y. (directeur scientifique) 2003. L'odyssée de l'espèce. Film de J. Malaterre. Coproduction France 3 - Transparences Productions - 17 juin Production - Pixcom RTBF - Mac Guff.

Corbin, L., Borel, D. et Camos, V. 2orza. Dissociation mémoire de travail/vitesse de traitement chez les enfants intellectuellement précoces au travers de deux études de cas. Enfance 4: 373-387.

Corbin, L., Moissenet, A. et Camos, V. 20I2b. Fonctionnement de la mémoire de travail chez des enfants présentant des difficultés scolaires. Développements II : 5-I2.

Cотте, P. 1987. Réflexions sur l'emploi des temps du passé en français et en anglais à la lumière de deux évolutions récentes du système verbal de l'anglais. Contrastes I4-i5: 89-16I.

Cotтe, P. 199. L'adverbialisation par l'effacement et par la condensation. Travaux linguistiques du Cerlico 4: IO4-I49.

Cотte, P. 2008. Expressivité secrète. Bulletin de la Société de stylistique anglaise 30: II-27. En ligne à l'adresse suivante : http://stylistique-anglaise.org/document.php?id= $=508$. 
Damazio, A.R. 2006. Remembering When. Scientific American I6 (I): 34-4I. En ligne à l'adresse suivante: http://www.srvhs.srvusd.kı2.ca.us/staff/teachers/Sperri/Articles/ Remebering time.pdf.

Davies, P. 2006. That Mysterious Flow. Scientific American I6 (I) : 6-II.

DeHAENE, S. 2006. Les bases cérébrales d'une acquisition culturelle: la lecture. In L'enfant précoce en marche vers l'adolescence (Actes du congrès de l'AFEP, 28 janvier 2006, université de Paris-Sorbonne). Paris: AFEP: 13-19.

Dehaene, S. 2008. Les neurones de la lecture. Canal académie. 20 janvier 2008. En ligne à l'adresse suivante: http://www.canalacademie.com/Les-Neurones-de-la-lecture.html.

Dehaene-Lambertz, G. 2006. L'organisation du cerveau du nourrisson pourrait-elle expliquer l'acquisition rapide de la langue maternelle? Communiqué de presse du CNRS en ligne à l'adresse suivante: http://www2.cnrs.fr/presse/communique/924.htm.

Dehaene-Lambertz, G. 2008. La renaissance de la parole. In P. PicQ et al. (éd.), La plus belle bistoire du langage. Paris: Seuil: $125-184$.

Desmarais, G. et al. 1997. Mémoire et apprentissage. Rapport de synthèse préliminaire, 24 septembre 1997. En ligne à l'adresse suivante: http://ecoles.cstrois-lacs.qc.ca/garcons/ recherche et littérature/MEMOIRE_ET_APPRENTISSAGE.doc.

Dietrich, R., Klein, W. et Noyau, C. 1995. The Acquisition of Temporality in a Second Language. Amsterdam - Philadelphie: J. Benjamins.

Droit-Volet, S. 2000. L'estimation du temps: perspective développementale. L'année psychologique Iоо (3): 443-464.

Droit-Volet, S. 200I. Les différentes facettes du temps. Enfances et psy 13: 26-40.

Droit-Volet, S. 2oir. Child and Time. In A. Vatakis et al. (éd.), Multidisciplinary Aspects of Time and Time Perception. Berlin - Heidelberg: Springer: I5I-I73.

Droit-Volet, S., Delgado, M. de L. et Rattat, A.-C. 2006. The Development of the Ability to Judge Time in Children. In J.R. Marrow (éd.), Focus on Child Psychology Research. New York: Nova Science Publishers: 8I-IO4.

FAYOL, M. 1982. Le plus-que-parfait. Étude génétique en compréhension et production chez l'enfant de quatre à dix ans. Archives de psychologie 50 (195) : 26I-283.

FERREIRO, E. 197I. Les relations temporelles dans le langage de l'enfant. Genève - Paris: Droz.

Ferreiro, E. et Sinclair, H. I97I. Temporal Relationships in Language. International Journal of Psychology 6 (I): 39-47.

Fortson, B.W. 2oIo. Indo-European Language and Culture. An Introduction. Blackwell textbooks in linguistics I9. Chichester: Wiley-Blackwell.

Fuchs, C. et al. 1978a. Études sur l'aspect: théorie et description. Essais sur le serbo-croate, le slovène, l'arabe marocain, le hausa, le japonais et le grec moderne. Paris: Université de Paris VII.

Fuchs, C. et al. (éd.) 1978b. Documentation et recherche en linguistique allemande contemporaine: Quelques aspects de l'aspect I6. Paris: Université de Paris VIII.

Gosselin, L. 1996. Sémantique de la temporalité en français. Un modèle calculatoire et cognitif du temps et de l'aspect. Champs linguistiques. Louvain-la-Neuve: Duculot. 
Gougenheim, G.R. et al. 1964. L'élaboration du français fondamental, Ir degré: étude sur l'établissement d'un vocabulaire et d'une grammaire de base. Paris : Didier.

Haudry, J. 1979. L'indo-européen. Que sais-je? 1798. Paris: PUF.

HowARD, M. 2002. L'acquisition des temps du passé en français par l'apprenant dit avancé: une approche lexicale. In E. LABEAU et P. LARrivéE (éd.), Les temps du passé français et leur enseignement. Cahiers chronos 9. Amsterdam: Rodopi : I8I-204.

IzARD, V. et al. 20II. Geometry as a Universal Mental Construction. In S. DehaEne et E.M. Brannon (éd.), Space, Time and Number in the Brain. Searching for the Foundations of Mathematical Thought. San Diego: Elsevier - Academic Press: 319-332.

KING, G. et al. 1995. Are English-Speaking SLI Children with Morpho-syntactic Deficits Impaired in Their Use of Locative-Contact and Causative Alternating Verbs? University of Reading Working Papers in Linguistics 2: 45-66.

KInG, K.A. 2006. Child Language Acquisition. In R.W. FAsold et J. Connor-Linton (éd.), An Introduction to Language and Linguistics. Cambridge: Cambridge University Press : 235-273.

KLEIN, W. 1994. Time in Language. Londres - New York: Routledge.

Knierim, J.J. 2007. The Matrix in Your Head. Scientific American Mind I8 (3): 42-48.

Kraft, U. 2007. Rhythm and Blues. Scientific American Mind I8 (3): 62-65.

Kurylowicz, J. 1977. Problèmes de linguistique indo-européenne. Wrocław: Polska Akademia Nauk.

Labeau, E. 2002. The Acquisition of French Past Tenses by Tutored Anglophone Advanced Learners: Is Aspect enough? Thèse de doctorat non publiée. Aston University.

LABEAU, E. 2004. Au-delà de l'hypothèse de l'aspect: le développement aspectuo-temporel dans l'interlangue d'apprenants avancés. In Acquisition, pratiques langagières, interactions et contacts - APLIC (Colloque international en sciences du langage, Paris, université Sorbonne Nouvelle, $25-26$ juin 2004). En ligne à l'adresse suivante: http://eprints.aston. ac.uk/7207/I/Au-delà_de_l'hypothèse_de_l'aspect_le_développement_du_temps_et_ de_l'aspect_dans_l'interlangue_d'apprenants_avancés.htm.

Labeau, E. 2005a. Line or Circle? The Process of Past Tenses Acquisition by Advanced Learners of French. In J. CoHEn et al. (éd.), ISB4: Proceedings of the 4th International Symposium on Bilingualism. Somerville: Cascadilla Press: I264-I275. En ligne à l'adresse suivante: http://www.lingref.com/isb/4/o99ISB4.PDF.

LABEAU, E. 2005b. Des «Temps modernes»: l'aspect suffit-il à la maîtrise des temps du passé dans les narrations écrites d'apprenants avancés? In A.L. MolendiJK et J.P. VET (éd.), Temporalité et attitude: structuration du discours et expression de la modalité. Cahiers chronos I2. Amsterdam: Rodopi : 203-218. En ligne à l'adresse suivante: http://eprints. aston.ac.uk/155I/I/Labeau_(Chronos_5).pdf.

Labelle, F. 2002. Point de vue et aspect en français et en anglais. In E. LABEAU et P. LARrivée (éd.), Les temps du passé français et leur enseignement. Cahiers chronos 9. Amsterdam: Rodopi : 72-9I.

Labelle, M. 1994. Acquisition de la valeur des temps du passé par les enfants francophones. Revue québécoise de linguistique 23 (I) : 99-I2I. 
LEONARD, L.B. 1989. Language Learnability and Specific Language Impairment in Children. Applied Psycholinguistics Io (2): 179-202.

LeOnARD, L.B. et al. 1992. Morphological Deficits in Children with Specific Language Impairment : The Status of Features in the Underlying Grammar. Language Acquisition $2(2): 15 \mathrm{I}-\mathrm{I} 79$.

Marchman, V. et Weismer, S.E. 1994. Patterns of Productivity in Children with SLI and NL: A Study of the English Past Tense. In Annual Meeting of the Symposium on Research in Child Language Disorders - SRCLD. Madison: University of Winconsin.

McClelland, J.L. et Patterson, K. 2002. Rules or Connections in Past-Tense Inflections: What does the Evidence Rule Out? Trends in Cognitive Sciences 6 (II) : 465-472.

Meisel, J. 1992. The Acquisition of Verb Placement: Functional Categories and V2 Phenomena in Language Acquisition. Dordrecht - Boston - Londres: Kluwer Academic Publishers.

Mora, G., Camos, V. et Baroulllet, P. 20io. L'utilisation des mécanismes de maintien de l'information verbale en mémoire de travail chez l'enfant de 8 ans. In $1 \sigma^{e}$ forum des Jeunes Chercheurs. Besançon. Support de communication en ligne à l'adresse suivante: http:// leadserv.u-bourgogne.fr/files/publications/ooo548-1-utilisation-des-mecanismes-demaintien-de-1-information-verbale-en-memoire-de-travail-chez-1-enfant-de-8-ans.pdf.

MüLLER, C. 1974. Les verbes les plus fréquents du français. Le français dans le monde $\mathrm{IO}_{3}$ : I3-17.

Myrill, J. 199i. Typological Text Analysis: Tense and Aspect in Creoles and Second Languages. In T. Huebner et C.A. Ferguson (éd.), Crosscurrents in Second Language Acquisition and Linguistic Theories. Amsterdam - Philadelphie: J. Benjamins: 93-I22.

Noyau, C. 200I. Typologie et dynamiques des langues: les études acquisitionnelles sur les processus de morphologisation temporelle. Linx: Invariants et variables dans les langues. Études typologiques 45: 177-I84.

Noyau, C. 2005. Le lexique verbal dans des restitutions orales de récits en français L2: verbes de base, flexibilité sémantique, granularité. In F. Grossmann, M.-A. Paveau et G. Petit (éd.), Didactique du lexique: langue, cognition, discours. Grenoble: ELLUG: $65-84$.

Noyau, C. 2008. Place des verbes dans le Français Fondamental, acquisition du lexique verbal en français langue seconde, et didactique du lexique. Le français dans le monde - Recherches et applications: Quel oral enseigner, cinquante ans après le français fondamental? 43: 87-IOO.

Oetring, J., Horohov, J.E. et Costanza, A.L. I995. Influences of Stem and Root Characteristics on Past Tense Marking: Evaluation of Children with SLI. In Annual Meeting of the Symposium on Research in Child Language Disorders - SRCLD. Madison: University of Winconsin.

O’Kelly, D. 1994. Du référent expérientiel au référent mental: pour une approche de la temporalité. Modèles linguistiques I5 (I) : 25-68.

Paprocka-Piotrowska, V. 2002. «Mais dans ce moment le chien est venu» ou comment les apprenants formels polophones s'approprient le système temporel du français. In E. Labeau et P. LARRIVÉE (éd.), Les temps du passé français et leur enseignement. Cahiers chronos 9. Amsterdam: Rodopi : 209-234. 
Paradis, J. et Crago, M. 200o. Tense and Temporality: A Comparison between Children Learning a Second Language and Children with SLI. Journal of Speech, Language, and Hearing Research 43 (4): 834-848.

Piaget, J. 1937. La construction du réel chez l'enfant. Neuchâtel - Paris: Delachaux et Niestlé. Piaget, J. 2006 [1926]. La représentation du monde chez l'enfant. Paris: PUF.

Pierce, A.E. 1992. Language Acquisition and Syntactic Theory: A Comparative Analysis of French and English Child Grammars. Dordrecht - Boston - Londres : Kluwer Academic Publishers.

Pinker, S. 199i. Rules of Language. Science 253 (5019): 530-535.

Pinker, S. 1999. Words and Rules. New York: Basic Books.

Pinker, S. 200I. Four Decades of Rules and Associations, or Whatever Happened to the Past Tense Debate? In E. Dupoux (éd.), Language, Brain and Cognitive Development: Essays in Honor of Jacques Mebler. Cambridge: MIT Press: 157-I79.

Planche, P. 1998. La construction des notions spatiales chez les enfants intellectuellement précoces, âgés de six à huit ans. Enfance 5I (2): I59-I7I.

Planche, P. 1999. Les stratégies de décentration spatio-cognitives chez les enfants intellectuellement précoces de 6 ans. Bulletin de psychologie 52-4 (442): 473-480.

Planche, P. 200o. Le fonctionnement et le développement cognitifs de l'enfant intellectuellement précoce. L'année psychologique Ioo (3): 503-525.

Ramscar, M. 2002. The Role of Meaning in Inflection: Why the Past Tense does not Require a Rule. Cognitive Psychology 45: 45-94.

Rice, M.L. et al. 2000. Acquisition of Irregular Past Tense by Children with Specific Language Impairment. Journal of Speech, Language, and Hearing Research 43 (5): II26-II45.

Rice, M.L. et Oetting, J.B. I993. Morphological Deficits of Children with SLI: Evaluation of Number Marking and Agreement. Journal of Speech, Language, and Hearing Research 36 (6) : I249-I257.

Rice, M.L. et WeXler, K. 1996. Toward Tense as a Clinical Marker of Specific Language Impairment in English-Speaking Children. Journal of Speech, Language, and Hearing Research 39 (6) : I239-1257.

Rizzi, L. 1993. Some Notes on Linguistic Theory and Language Development: The Case of Root Infinitives. Language Acquisition 3 (4): 371-393.

Robison, R.E. 1990. The Primacy of Aspect: Aspectual Marking in English Interlanguage. Studies in Second Language Acquisition I2 (3): 315-330.

Rousseau, A. 20Io. La problématique du temps. Réflexions méthodologiques à partir des paramètres des langues naturelles. In C. DouAy (éd.), Système et chronologie. Rivages linguistiques. Rennes: Presses universitaires de Rennes: 17-39.

Roussel, E. 2007a. Point de vue neurolinguistique sur des réalisations linguistiques. Anglophonia 22: 16I-178.

Roussel, E. 2007b. Une propriété pragmatique du past perfect. Corela 5 (2). En ligne à l'adresse suivante: http://corela.edel.univ-poitiers.fr/index.php?id=397.

Roussel, E. 2009. Regards croisés sur la temporalité. Monographie non publiée. 
Rumelhart, D.E. et McClelland, J.L. 1986. On Learning Past Tenses of English Verbs. In D.E. Rumelhart et J.L. McClelland (éd.), Parallel Distributed Processing. Cambridge: MIT Press. Vol. 2, Psychological and Biological Models: 216-27I.

SAbEAU-Jouannet, E. 1977. L'expression de l'organisation spatiale et temporelle, son évolution chez l'enfant de 2 à 5 ans. In F. Françors et al. (éd.), La syntaxe de l'enfant avant 5 ans. Paris: Larousse: 193-204.

Sergent, B. 2005. Les Indo-Européens. Histoire, langues, mythes. Paris: Payot et Rivages.

SiAUD-F ACCHIN, J. 20I2. L'enfant surdoué: l'aider à grandir, l'aider à réussir. Paris: O. Jacob.

SLoBIN, D.I. 197I. The Ontogenesis of Grammar: A Theoretical Symposium. New York - Londres: Academic Press.

Slobin, D.I. 198I. The Origins of Grammatical Encoding of Events. In W. Deutsch (éd.), The Child's Construction of Language. New York - Londres: Academic Press: I85-199.

SLOBIN, D.I. 1985. Crosslinguistic Evidence for the Language-Making Capacity. In D.I. Slobin (éd.), The Crosslinguistic Study of Language Acquisition. Hillsdale - Londres: L. Erlbaum. Vol. 2, Theoritical Issues: $1157^{-1256 .}$

SLobin, D.I. I99I. Learning to Think for Speaking: Native Language, Cognition and Rhetorical Style. Pragmatics I (I): 7-25.

SLOBIN, D.I. 1996. The Origins of Grammaticizable Notions: Beyond the Individual Mind. In D.I. Slobin (éd.), The Crosslinguistic Study of Language Acquisition. Hillsdale - Londres: L. Erlbaum. Vol. 5, Expanding the contexts: 265-323.

SMITH, C.S. 1980. The Acquisition of Time Talk: Relations between Child and Adult Grammars. Journal of Child Language 7 (2): 263-278.

Smith, C.S. et WeIst, R.M. 1987. On the Temporal Contour of Child Language: A Reply to Rispoli and Bloom. Journal of Child Language I4 (2): 387-392.

SPELKE, E.S. 20II. Natural Number and Natural Geometry. In S. DehaEnE et E.M. BRANnon (éd.), Space, Time and Number in the Brain. Searching for the Foundations of Mathematical Thought. San Diego: Elsevier - Academic Press: 287-317.

SweEtser, E. et Nunez, R. 2006. With the Future behind Them: Convergent Evidence from Aymara Language and Gesture in the Crosslinguistic Comparison of Spatial Construals of Time. Cognitive Science 30 (3): 40I-450.

Tomasello, M. 200o. First Steps toward a Usage-Based Theory of Language Acquisition. Cognitive Linguistics II (I-2): 6I-82.

Tomblin, B. 1994. Family and Twin Studies of Language Impairment. In American Association for the Advancement of Science Annual Meeting. San Francisco.

Ullman, M.T. 2006. Language and the Brain. In R.W. FAsold et J. Connor-Linton (éd.), An Introduction to Language and Linguistics. Cambridge: Cambridge University Press : $235^{-2} 273$.

Ullman, M.T. et Gopnik, M. 1994. The Production of Inflectional Morphology in Hereditary Specific Language Impairment. McGill Working Papers in Linguistics: Linguistic Aspects of Familial Language Impairment Io (I-2): 8I-II8.

VANDENPlas-HolPER, C. 1975. La compréhension d'expressions verbales de la succession temporelle par des enfants de 6 à II ans. Revue française de pédagogie 3I : 4-I5. 
Vet, C. 20ir. L'aspect grammatical en français. In F. Neveu, P. Blumenthal et N. Le Querler (éd.), Au commencement était le verbe: syntaxe, sémantique et cognition. Mélanges en l'bonneur du Professeur Jacques François. Berne - Berlin - Bruxelles: P. Lang: 443-465.

VINCENT, J.-D. 2007. Voyage extraordinaire au centre du cerveau. Paris: O. Jacob.

Weist, R.M. 1986. Tense and Aspect: Temporal Systems in Child Language. In P.J. Fletcher et M. Garman (éd.), Language Acquisition: Studies in First Language Development. Cambridge: Cambridge University Press : 356-374.

Weist, R.M. 1989. Time Concepts in Language and Thought: Filling the Piagetian Void from Two to Five Years. In I. Levin et D. ZAKaY (éd.), Time and Human Cognition: A Life-Span Perspective. Amsterdam - New York - Oxford: North-Holland: 63-II8.

WRIGHT, K. 2006. Times of Our Lives. Scientific American I6 (I) : 26-33. 